\title{
Generic Markov Model of the Contention Access Period of IEEE 802.15.4 MAC Layer
}

\author{
Joaquín Recas ${ }^{\ddagger}$, Nadia Khaled*, Alberto A. Del Barrio ${ }^{\ddagger}$ and Román \\ Hermida ${ }^{\ddagger}$ \\ ${ }^{\ddagger}$ Department of Computer Architecture and Automation, Computer Science Faculty, \\ Complutense University of Madrid, José Santesmases s $\backslash n$, 28040 Madrid, Spain \\ ${ }^{*}$ Bioanalytical Science Department, Nestec Ltd, Nestle Research Center, \\ Vers-chez-les-Blanc, P.O. Box 44, 1000 Lausanne 26.
}

\begin{abstract}
The IEEE-802.15.4 standard is poised to become the global standard for low data rate, low energy consumption Wireless Sensor Networks. By assigning the same sets of contention access parameters for all data frames and nodes, the Contention Access Period (CAP) of the slotted IEEE-802.15.4 currently provides an even channel access functionality and no service differentiation. However, some applications may require service differentiation and traffic prioritization support to accommodate high-priority traffic (e.g., alarms). In order to simulate a scenario in which different sets of access parameters for different node classes can be configured, this paper develops a Markov-chainbased model of the CAP of the IEEE-802.15.4-MAC. Our Markov model can be used to evaluate the impact of mixing node classes in important factors like the throughput, energy consumption, probability of delivery and the packet latency. The model has been used to provide traffic differentiation in a high saturation scenario in which a set of nodes can be configured to increase $76 \%$ the probability of sending a packet and reduce $58 \%$ latency, with a $69 \%$ energy penalty, in comparison with a standard scenario. The accuracy of the Markov model is validated by extensive $n s-2$ simulations.
\end{abstract}

*This work was supported in part by the Spanish Government Research Grant TIN200800508

Email address: recas@ucm.es, nadia.khaled@rdls.nestle.com, abarriog@ucm.es, rhermida@dacya.ucm.es (Joaquín Recas ${ }^{\ddagger}$, Nadia Khaled*, Alberto A. Del Barrio ${ }^{\ddagger}$ and Román Hermida ${ }^{\ddagger}$ ) 
Keywords:

Markov Chain, IEEE 802.15.4, Media Access Control, Quality of Service, Throughput, Wireless Sensor Networks, Energy Efficiency

\section{Introduction}

Recent advances in microelectronics have gone a long way towards the miniaturization and power efficiency of processing elements, radio transceivers and sensing structures of a large array of physical and biological phenomena. Capitalizing on these advances, unprecedented academic and commercial effort has gone into designing and deploying low-cost, low-power wireless sensor networks (WSNs) for a wealth of application areas, such as environment monitoring, industrial process surveillance, home automation and personal health monitoring. To support this effort, the IEEE 802.15.4 standard was devised, which specifies the physical (PHY) and medium access control (MAC) layers of such wireless sensor networks.

Since its ratification, the IEEE 802.15.4 MAC has received much interest to assess its throughput and energy performance. In particular, its beaconenabled mode featuring a novel slotted access protocol in its contention access period (CAP) has spurred most attention ${ }^{1}$. Many preliminary simulation studies $[2,3]$ have been conducted, and several accurate analytical models have been introduced. These analytical models follow two main approaches. The first approach is inspired from the seminal work of Bianchi on the analysis of IEEE 802.11 [4], and is based on modelling the behavior of a sensing node using a Markov chain. The most relevant contributions along this approach are [5], which adapts Bianchi's model to the specific contention access procedure of the slotted IEEE 802.15.4 CAP under unacknowledged saturated traffic conditions, [6] which corrects the analysis in [5] and extends it to include acknowledgment transmission, and [7] which proposes a more complex model reflecting additionally the superframe structure under unsaturated traffic conditions. A final relevant contribution, upon which our work is based, is the work of Ramachandran et al. [8] which develops a framework based on two Markov chains; the Node-State chain to determine the frac-

\footnotetext{
${ }^{1}$ The alternative ad-hoc non-beacon-enabled mode uses the non-slotted CSMA with collision avoidance mechanism, which is extensively studied in the literature and whose performance is well understood [1].
} 
tion of time a node spends in different states and the Channel-State chain to calculate the throughput of the network. This choice is motivated by the demonstrated accuracy of this model w.r.t. $n s-2$ simulations, as well as its simplicity and amenability for extension. The second approach to analytically model the IEEE 802.15.4 CAP, alternatively models the contention access mechanism as an embedded Markov renewal process and performs a fixed-point analysis to calculate the throughput of the network [9].

By assigning the same sets of contention access parameters for all data frames and nodes, the contention access period (CAP) of the slotted IEEE 802.15.4 medium access control (MAC) currently provides a priority-independent channel access functionality and no service differentiation. However, several wireless sensor network applications may require service differentiation and traffic prioritization support to accommodate delay sensitive traffic (e.g., alarms or emergency alerts in wireless body sensor networks) or prioritize high relevance/reliability messages throughout a large-scale sensor network.

Unlike [10], where only the Contention Window (CW) parameter is analysed, this work studies the impact of several parameters besides the Contention Window, namely: the number of backoff stages $\left(M_{b o}\right)$ and the probability of backing off before sensing the channel (related to macMinBE and macMaxBE), the behavior of the WSN in terms of the WSN total throughput, the energy consumption, the probability of delivery and the packet latency. The meaning of these parameters will be explained in the following sections. Different sets of nodes can be simulated, each with its specific values of access parameters, following the example of the IEEE 802.11e quality-of-service (QoS)-aware extension of the original IEEE 802.11 WLAN MAC. More specifically, this paper extends the Markov-chain-based analytical model of the IEEE 802.15.4 CAP in [8] in the presence of mixed sets of nodes with shared access parameters.

To the best of our knowledge, no such model exists for the IEEE 802.15.4 CAP with service differentiation. As a matter of fact, the only tentative model of differentiated access in the IEEE 802.15.4 MAC was presented in [11], and is not mathematically sound as it incorrectly assumes that the packet sending probability is independent from node to node, instead of the channel sensing probability. Finally, a similar accurate model does exist for the IEEE 802.11e enhanced distributed coordination function [12], but is not directly applicable to the IEEE 802.15.4 CAP due to their different contention access procedures. 
The objectives of this work are the following:

1. To propose a Markov-chain-based analytical model of a generic IEEE 802.15.4 node in which the main contention access parameters can be tuned.

2. To propose a Markov-chain-based analytical model of the wireless communication channel shared by different types of nodes.

3. To define a set of metrics to evaluate the impact of the contention parameters in the network.

4. To validate the model using $n s-2$ discrete event network simulator [13].

5. To obtain a set of contention access parameters that allow to implement service differentiation.

6. To provide a tool able to comply with the previous items. Each case of study provided in the paper can be reproduced using the code for Octave/Matlab available at the following repository [14]

The rest of the paper is organized as follows. Section 2 reviews the slotted IEEE 802.15.4 MAC protocol, and the underlying slotted CSMA/CA algorithm. Section 3 introduces our extension of the state-of-the-art IEEE 802.15.4 Markov model to allow CAP parameters modification and in Section 4 a set of evaluation metrics are defined. This extended Markov model is validated by extensive $\boldsymbol{n s}$ - 2 simulations and used to evaluate the performance of a contention-based service differentiation strategy in Section 5. Finally, the paper is concluded in Section 6.

\section{Overview of the Slotted IEEE 802.15.4 CSMA/CA Channel Ac- cess Algorithm}

In this Section, we briefly review the MAC protocol of the slotted mode of the IEEE 802.15.4 standard, which specifies the time structure, default parameter settings and channel access procedure for beacon-enabled PANs (Personal Area Networks). A more comprehensive description of the standard may be found in $[15,2]$.

In the slotted mode, a coordinator periodically transmits a beacon to identify its PAN, synchronize the nodes associated with it, and delimit the superframe time structure that organizes the communication in the network. The superframe begins with a beacon, followed by an active and an optional inactive period, see Figure 1. All communication takes place during 


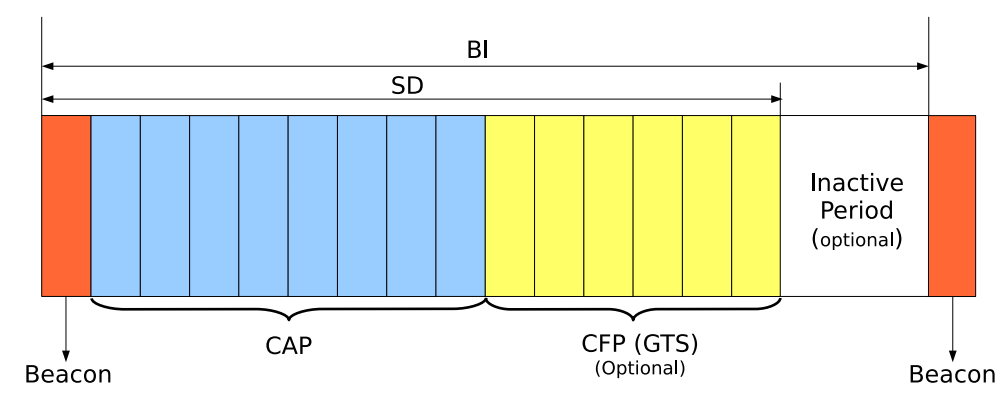

Figure 1: IEEE 802.15.4 superframe structure in the beacon-enabled mode

the active period, whereas all nodes may be powered down during the inactive period to conserve energy. The active period of a superframe consists of a contention access period (CAP) and an optional contention-free period (CFP). In the CFP, the PAN coordinator controls the channel access by assigning guaranteed time slots (GTS) to those nodes that request the access. The actual GTS assignment and the corresponding CFP performance are determined by the GTS scheduling algorithm used by the PAN coordinator, which is openly described in the standard. However, GTS may not be the best choice while considering a large amount of nodes [16, 17]. Statically allocating slots may waste bandwidth that could be used during the contention period. This effect is more noticeable in scenarios as ours, where there are only 16 time slots between beacons. Thereby, under these conditions it is hard for GTS to guarantee the CFP frame transmissions. Alternatively, the CAP allows nodes to access the channel in a distributed fashion through a non-persistent slotted CSMA/CA algorithm, which is also completely specified in the standard as follows.

In the slotted CAP, all nodes are synchronized and can start the transmission only at the beginning of segmented time slots, called backoff slots. The duration of one slot is aUnitBackoffPeriod (the default value is 20 symbols, i.e., $320 \mu \mathrm{s}[15])$. When a node has a new data frame awaiting for transmission at the MAC buffer, see Figure 2, it first initializes the three relevant contention parameters, namely the number of random backoff stages experienced $(\boldsymbol{N B} \in \mathbb{N})$ to 0 , the current backoff exponent $(\boldsymbol{B} \boldsymbol{E} \in \mathbb{N})$ to macMinBE (default value 3 ) and the contention window $(C W \in \mathbb{N}$ with a default value 2). Then, it selects a backoff counter $(B C \in \mathbb{N})$ value uniformly from the range $\left[0 \leq B C \leq 2^{B E}-1\right]$. This backoff counter value is decremented 


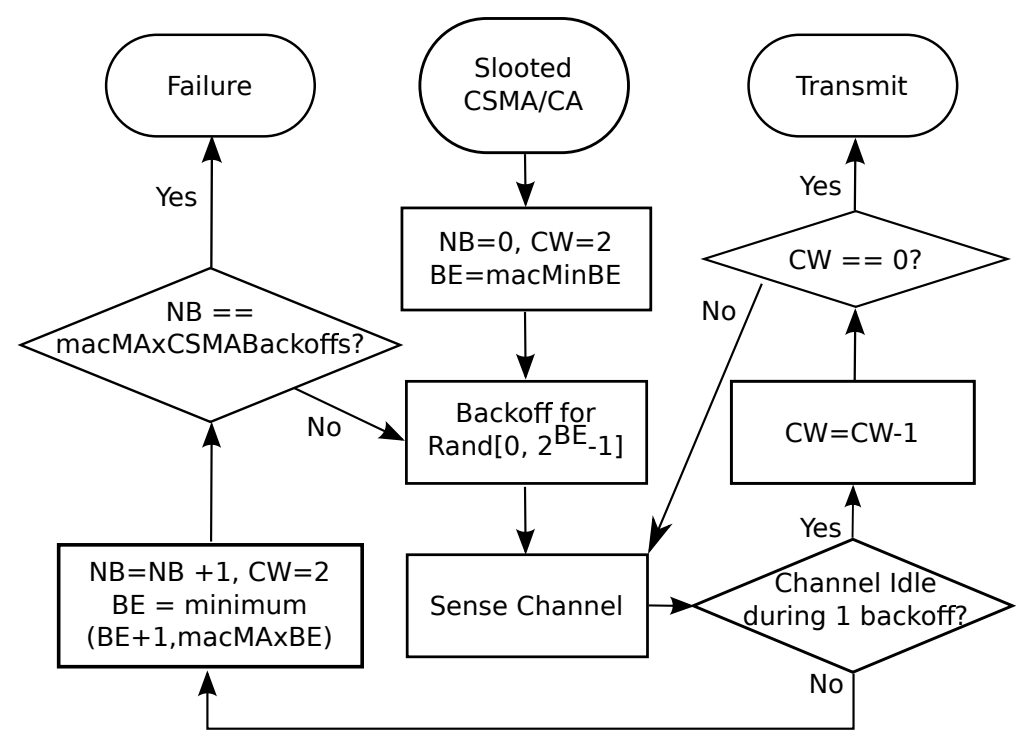

Figure 2: The slotted CSMA/CA algorithm for IEEE 802.15.4

by one for each backoff slot regardless of the channel state. As soon as the backoff counter reaches zero, the node performs carrier sensing that consists of $C W$ consecutive clear channel assessments (CCA). If the channel is sensed idle, $C W$ is decremented by one and the node performs the following CCA at the beginning of the next backoff slot. Only when the channel is assessed idle during $C W$ consecutive CCAs, the node will start transmission in the next backoff slot. Otherwise, the node will enter the next backoff stage, i.e., it will increase the values of $N B$ and $B E$ by one, will reset $C W$ to its initial value and will draw a new random number of backoff slots from the updated window $\left[0 \leq B C \leq 2^{B E}-1\right]$ to delay the channel for being sensed again. This procedure is repeated until the frame is transmitted, or a channel access failure is declared. The latter occurs when $N B$ reaches the maximum number of random backoff stages allowed, macMaxCSMABackoffs (default value $=4$ ). Note that $B E$ shall not be incremented beyond its maximum value, defined by $\operatorname{macMaxBE}$ (default value $=5$ ); beyond this value, $\mathrm{BE}$ is frozen to $\operatorname{macMaxBE}$.

CSMA/CA algorithm, which is also completely specified in the standard as follows. 


\section{Markov-chain-based analytical Model}

As aforementioned, this section extends the analytical model of the IEEE 802.15.4 CAP in [8] in the presence of different sets of nodes. Our proposal extends this work and develops a node-state chain for each set of nodes that share the same contention access parameters, as well as a channel-state chain. All other contention access parameters are set to the default values [15]. We consider a single-hop wireless sensor network consisting of a Coordinator and different sets of nodes, grouped by classes. This is a reasonable assumption, since nodes use to be close inside a WSN $[18,19,8]$. The analytical model will be presented using a case of study composed of three different classes of nodes and a coordinator, and extended for a general scenario. A general classn group consists on $M_{n}$ nodes that use the same contention parameters, each node will be addressed by a class-n node. Furthermore, we consider a star topology and uplink data transmission scenario without acknowledgement, as other authors do [20, 21, 22].

\subsection{Model assumptions and approximations}

As an extension of the analytical model introduced in [8], the present work is based on the same assumptions and approximations whose accuracy have been validated therein. For self-containment, we summarize the most relevant assumptions and approximations:

1. All nodes are time-synchronized with the coordinator's beacon and are within the transmission range of each other (no hidden-node problem). We must pinpoint that this synchronization should be produced in such a manner that the clock skew is smaller than a backoff slot. In this way, Markov chain transitions are correct, as in order to detect whether the channel is free or busy we devote a whole backoff.

2. The nodes are not allowed to sleep and the entire superframe duration is active and set to be long enough. The reason for this is to accurately approximate the CSMA/CA scheme as non-persistent CSMA, and neglect the effect of finite superframe duration [3].

3. All of the active periods of the superframe are allocated to the CAP.

4. Only direct transmission is considered and the PAN coordinator is configured to do not acknowledge the reception of the packets which have a fixed duration of $N$ slots for all nodes. 
5. Initially, the nodes have no packets to transmit and the packets arrive for transmission according to a Poisson arrival rate of $\lambda \in \mathbb{R}^{+}$ packets per packet duration with $[0 \leq \lambda \leq 1]$. Also, no packet buffering is allowed while a node is transmitting or has already initiated the CSMA/CA scheme.

6. The uniform distribution specified in the IEEE 802.15.4 standard (to draw the number of backoff slots a node has to wait at each backoff stage of the contention access procedure) is replaced with a geometric distribution of the same mean number of backoff slots so that the backoff procedure remains memoryless. In order to maintain the mandatory Markov memorylessness feature, this distribution must be used [4].

Notation: For notational clarity, all probabilities associated with channel states have a superscript ' $c$ ' (e.g., $p_{i}^{c}$ is the probability for the channel of being idle), and those associated with a class-n node state have a superscript ' $n$ ' (e.g., $p_{t}^{n}$ is the probability for a generic class-n node of begin a transmission in a generic backoff slot). Also, for the sake of clarity in the figures, the complementary probability $1-p$ has been substituted by $\bar{p}\left(\right.$ e.g., $\bar{p}_{i}^{c} \equiv 1-p_{i}^{c}$ ).

\subsection{Case of Study}

The CSMA/CA mechanism of each class-n node is modelled by means of a corresponding discrete-time Markov chain, as proposed by $[8,10]$, but we will extend the Markov chain in order to describe a more complete scenario. Not only a $C W$ of 1 and 2 is allowed, as in [10], but a set of different contention parameters are allowed for each class of nodes, with no restriction about its values. In our work we will study:

- $C W$ : the Contention Window assigned to the node, that is the number of consecutive time slots that the node has to sense the idle channel before transmitting.

- macMaxCSMABackoffs: the number of permitted random backoff stages, that will be denoted as $M_{b o}$.

- macMinBE: initial backoff exponent.

- macMaxBE: maximum backoff exponent.

The generic model will be presented using a simple scenario with one Coordinator and three different groups of nodes, i.e., class-n1, class-n2 and 


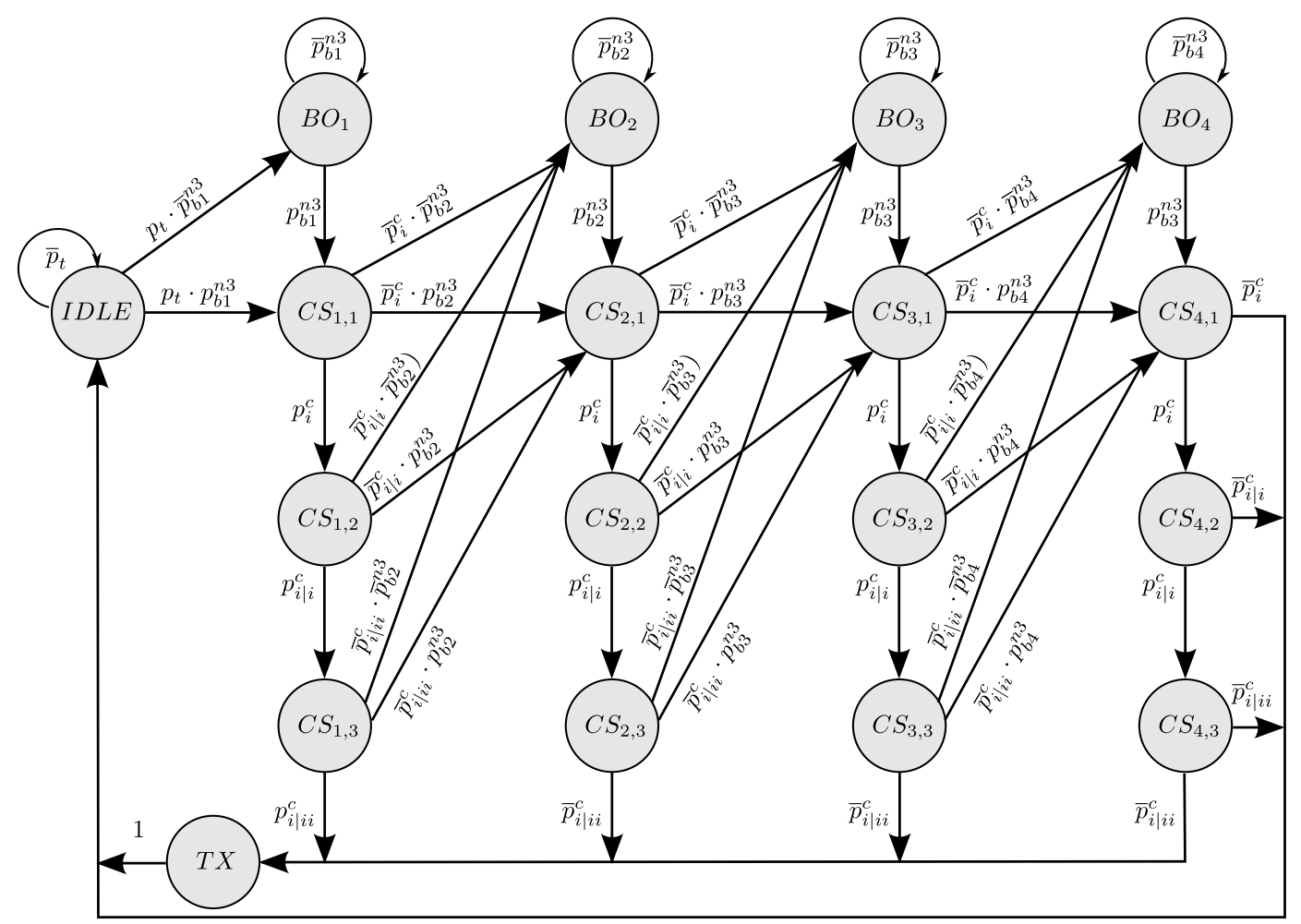

Figure 3: Node-State Markov chain model for a class-n3 node with $C W^{n 3}=3$ and $M_{b o}^{n 3}=$ 4

class-n3 groups with 4 nodes per group. Each group has a different set of contention parameters, but share the same packet duration of $N=10$ slots and $\lambda=0.9$ transmission rate, as introduced in 3.1:

- class-n1: $C W^{n 1}=2, M_{b o}^{n 1}=4, \operatorname{macMin} B E^{n 1}=3$ and $\operatorname{macMax} B E^{n 1}=5$. These are the default parameters for 802.15.4 CAP.

- class-n2: $C W^{n 2}=2, M_{b o}^{n 2}=3, \operatorname{macMin} B E^{n 2}=3$ and $\operatorname{macMax} B E^{n 2}=5$

- class-n3: $C W^{n 3}=3, M_{b o}^{n 3}=4, \operatorname{macMinB} E^{n 3}=0$ and $\operatorname{macMax} B E^{n 3}=5$

\subsection{Node-State models}

Figure 3 shows an Embedded Markov chain model for the class-n3 nodes with $C W^{n 3}=3$ and $M_{b o}^{n 3}=4$. Initially, a node is in the IDLE state and 
would remain in that state until it receives one or more packets for transmission during a backoff slot, with probability:

$$
p_{t}=1-e^{-\lambda / N}
$$

since every node receives packets of $N$ backoff slots of duration arriving with a Poisson arrival rate of $\lambda$ packets per packet duration, and therefore, the probability that no packet is received during a backoff slot is $e^{-\lambda / N}$. Once the class-n node has a packet to transmit, it would spend in the first backoff state, $B O_{1}$, a random number of backoff slots $X_{1}$ geometrically distributed according to $P\left[X_{1}=k\right]=\left(1-p_{b 1}^{n}\right)^{k} \cdot p_{b 1}^{n}$, in which $p_{b 1}^{n}$ is the probability of leaving the backoff stage 1 for a class-n node. The 802.15.4 standard specifies that this value should be drawn from a uniform distribution between 0 and $2^{B E^{n}}-1$, with an initial value of $B E^{n}=\operatorname{macMin} B E^{n}$ and increasing $B E^{n}$ on each backoff stage until the maximum value defined by $\operatorname{macMax} B E^{n}$ (i.e for a class-n1 node, the $B E^{n 1}$ values for $B O_{1}$ to $B O_{4}$ will be vector $\left.\mathbf{B E}^{n 1}=\left[\begin{array}{llll}3 & 4 & 5 & 5\end{array}\right]\right)$. In general, we adopt the notation $B O_{j}$ to represent the $j^{\text {th }}$ random backoff stage (note that $1 \leq j \leq M_{b o}^{n}$ ). As mentioned in Section 3.1, a geometrical distribution has been used to keep the procedure memoryless, and hence, in order to maintain the same mean number of backoff slots to be selected for a generic backoff stage $B O_{j}$, we have:

$$
p_{b j}^{n}=\frac{1}{1+\left(2^{\mathbf{B E}^{\mathbf{n}}(j)}-1\right) / 2},
$$

that will generate vector $\mathbf{p}_{b}^{n 1}=[1 / 4.5,1 / 8.51 / 16.5,1 / 16.5]$. In general, for a class-n node we will denote this vector as $\mathbf{p}_{b}^{n}$.

Upon leaving $B O_{1}$, the node moves to state $C S_{1,1}$ with a probability of $p_{b 1}^{n}$, which corresponds to performing the first CCA. If the channel is found busy in the first CCA, which occurs with the probability $\bar{p}_{i}^{c}$, the node will enter in the next backoff stage $\mathrm{BO}_{2}$ or second CCA. If the channel is found idle, the node will enter into $C S_{1,2}$. If the channel is again found idle for the second consecutive time, an event characterized by the conditional probability $p_{i \mid i}^{c}$, the node will enter in the next stage $C S_{1,3}$. We use the notation $C S_{j, k}$ to denote the $k^{\text {th }}$ carrier sense attempt after the $j^{\text {th }}$ random backoff stage of a class-n node $\left(1 \leq j \leq M_{b o}^{n}\right.$ and $\left.1 \leq k \leq C W^{n}\right)$. For a generic node, the channel has to be sensed idle $C W^{n}$ consecutive backoff slots, and will move from $C S_{j, C W^{n}-1}$ into state $C S_{j, C W^{n}}$ with a conditional probability $p_{i \mid\left(C W^{n}-2\right) i}^{c}$, that means that the channel has been sensed idle given that has 
been idle $C W^{n}-2$ consecutive slots. In this notation, the subindex between parenthesis represents the number of consecutive slots the channel has been idle, for example $p_{i \mid i i}^{c} \equiv p_{i \mid(2) i}^{c}$ (i.e., the channel is sensed idle giving that it has been idle for the previous two consecutive backoff slots) and $p_{i}^{c} \equiv p_{i \mid(0) i}^{c}$ (i.e., the channel is sensed idle with no assumptions about previous states).

The CCA process continues until the class-n node or fails, the maximum number of allowed random backoff stages is reached and a channel access failure is declared, or succeeds and enters in the transmit state $T X$, spending therein $N$ backoff slots and going back to the IDLE state with an associated probability of 1 . Let $p_{t}^{n}$ denote the probability of a class-n node begins transmission in a generic backoff slot. According to Figure 3, a node will begin a transmission in the next backoff slot if, being in the $C S_{j, C W^{n}}$ state, it senses the channel idle. The channel access probability $p_{t}^{n}$ of a class-n is given by:

$$
p_{t}^{n}=\frac{\sum_{j=1}^{M_{b o}^{n}} \pi_{C S j, c w}^{n}}{\sum N d S t^{n}} \cdot p_{i \mid\left(C W^{n}-1\right) i}^{c},
$$

where $\pi_{C S j, c w}^{n}$ is the long-run proportions of transitions into state $C S_{j, C W^{n}}$ of a class-n node and $\sum N d S t^{n}$ is the summation of all possible node states. The steady state occupancy of the states $I D L E, B O_{j}, C S_{j, k}$ and $T X$ for a generic class-n node, denoted as $\pi_{I D L E}^{n}, \pi_{B O j}^{n}, \pi_{C S j, k}^{n}$ and $\pi_{T X}^{n}$, can be obtained by solving the Markov chain steady state equations shown in Appendix A. The summation of all possible channel states can be obtained from:

$$
\sum N d S t^{n}=\pi_{I D L E}^{n}+\sum_{j=1}^{M_{b o}^{n}} \sum_{k=1}^{C W^{n}} \pi_{C S j, k}^{n}+\sum_{j=1}^{M_{b o}^{n}} \pi_{B O j}^{n}+N \cdot \pi_{T X}^{n} .
$$

To completely determine the behavior of a node at the steady state, the conditional probabilities $p_{i \mid(j) i}$ must be computed. If we knew the probability for the channel of being idle a certain amount of slots, $p_{(j) i}$ in case of $j$ consecutive slots, we could derive the conditional probability using the following expression:

$$
p_{(j+1) i}^{c}=p_{(j) i}^{c} \cdot p_{i \mid(j) i}^{c} .
$$

Furthermore, for a packet length common to all the nodes and equal to $N$, the conditional probability $p_{i \mid i}$ can be computed as follows [8]: 


$$
p_{i \mid i}^{c}=\frac{N \cdot p_{i}^{c}-1+p_{i}^{c}}{N \cdot p_{i}^{c}} .
$$

Moreover, the conditional probability for a class-n node to start a transmission knowing that the channel has been idle at least $m$ consecutive slots, $p_{t \mid(m) i}^{n}$ should be computed in order to solve the channel Markov chain:

$$
p_{t \mid(m) i}^{n}=\frac{p_{t}^{n}}{p_{(m) i}^{c}} .
$$

In the following section, the channel will be modelled as a Markov chain and the probabilities for the channel of being idle an arbitrary number of slots will be obtained.

\subsection{Channel-State model}

Continuing with the previous scenario described in Section 3.2 with three different groups of nodes, the channel behavior can be described using the discrete-time Markov chain of Figure 4, which is constructed as follows. We define three different types of states: channel Idle, channel collision or Failure and Success states. The Idle states are divided into Busy - Idle states denoted by $B I_{j}$ and an Idle $-C W_{m}$ state (in which $C W_{m}$ is the maximum value of $C W$ for the set of nodes present in the network, that is 3 in the case of study), denoted by $I_{3} . B I_{j}$ corresponds to the channel having been idle exactly $j$ consecutive backoffs, with $1 \leq j \leq C W_{m}-1$ for the generic scenario, preceded by a channel busy backoff slot due to either Success or Failure. The Idle $-C W_{m}$ state corresponds with a state in which the channel has been idle for the past $C W_{m}$ backoff slots but could have been more. The Failure state, denoted by $F$, corresponds with a collision on the channel of two or more packets, and the dwelling time is equal to the packet length, $N$ backoff slots. Finally, the Sucess state corresponds with a state in which only one node starts the communication in the current backoff stage, and therefore the node is able to successfully transmit the data packet. The Success states have also a dwelling time of $N$ backoff slots, and we use the notation $S_{n}$, that represents a node of the class-n group sending a packet.

In an Idle state three different events can occur: the channel is idle in the next backoff slot, a packet is sent successfully or a collision occurs. The transition probability, $\alpha_{j}$, represents the probability that no node in the network begins a transmission given that the channel has been idle during $j$ previous slots, and can be computed as follows for a generic scenario: 


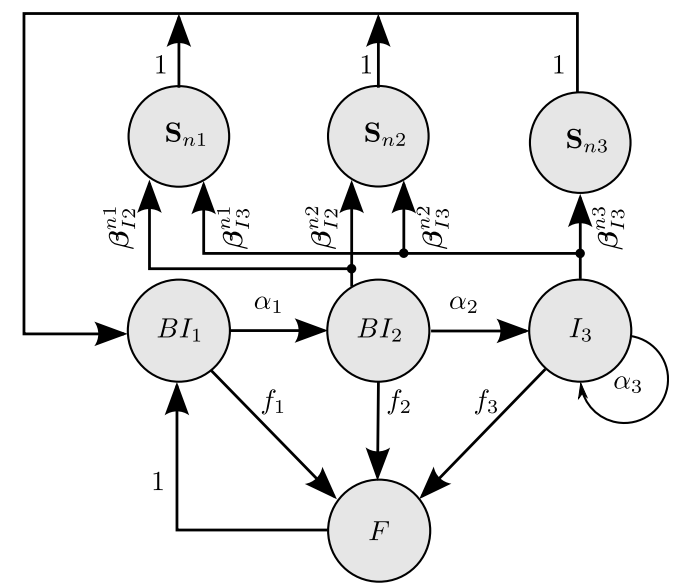

Figure 4: Channel-State Markov chain for the study case

$$
\alpha_{j}=\prod_{x=1}^{\infty} \prod_{m=1}^{j}\left(\bar{p}_{t \mid(m) i}^{n x}\right)^{M_{n x}},
$$

where $M_{n x}$ is the number of class-nx nodes in the network and $p_{t \mid(m) i}^{n x}$ is defined in eqn. 7. It will be denoted as vector $\boldsymbol{\alpha}$. It should be noticed that the transmission probabilities for the case of study $p_{t \mid(1) i}^{n 1}, p_{t \mid(1) i}^{n 2}$ and $p_{t \mid(1) i}^{n 3}$ are necessary equal to 0 , because none of these sets of nodes can begin a transmission when only one backoff slot has been idle, and therefore $\alpha_{1}=$ 1. In case of only two previous idle states, class-n 3 nodes can not begin a transmission (as can be seen in Figure 4), $p_{t \mid(2) i}^{n 3}=0$.

The transition probabilities form an Idle state to a $S_{n x}$ state is characterized by matrix $\boldsymbol{\beta}$. Each component will be denoted as $\beta_{I j}^{n x}$, where the super-index $n x$ represents the destination Success group and $I j$ represents the source index of the Idle group. These probabilities represent the situation in which only one node of class-nx succeeds in transmitting a packet while the other classes that are allowed to transmit, i.e., that have $C W \geq j$, do not attempt to transmit, and is given as follows: 


$$
\beta_{I j}^{n x}=\left\{\begin{array}{cl}
p_{t \mid\left(C W^{n x}\right) i}^{n x} \cdot M_{n x} \cdot\left(\bar{p}_{t \mid\left(C W^{n x}\right) i}^{n x}\right)^{M_{n x}-1} \cdot & \\
\prod_{\substack{y=1 \\
y \neq x}}^{\infty}\left(\bar{p}_{t \mid\left(C W^{n y}\right) i}^{n y}\right)^{M_{n y}} & \text { if } C W^{n x} \geq j \\
0 & \text { otherwise } .
\end{array}\right.
$$

The transition probability, $f_{j}$, for which the channel goes from an Idle state to $F_{j}$, is the probability that, while the channel being idle, at least two nodes, whatever their class, begin the transmission. This probability can be obtained knowing that the sum of the transition probabilities out of a state has to be equal to one:

$$
f_{j}=1-\alpha_{j}-\sum_{x=1}^{\infty} \beta_{I j}^{n x} .
$$

The steady state occupancy of the states $B I_{j}, I_{C W_{m}}, F$ and $S_{n x}$ for the channel, denoted as $\pi_{B I j}^{c}, \pi_{I c w}^{c}, \pi_{F}^{c}$ and $\pi_{S n x}^{c}$ can be obtained by solving the Markov chain steady state equations shown in Appendix B. Once the channel Markov chain has been solved, the steady state probabilities $p_{b,(j) i}^{c}$, that is the probability of $j$ consecutive idle periods after a busy slot, and $p_{\left(C W_{m}\right) i}^{c}$, the probability of $C W_{m}$ consecutive idle states, can be obtained:

$$
\begin{gathered}
p_{b,(j) i}^{c}=\frac{\pi_{B I j}^{c}}{\sum C h S t}, \\
p_{(C W m) i}^{c}=\frac{\pi_{I c w}^{c}}{\sum C h S t},
\end{gathered}
$$

where $\sum C h S t$ is the summation of all possible channel states and can be obtained from:

$$
\sum C h S t=\sum_{j=1}^{C W_{m}-1} \pi_{B I j}^{c}+\pi_{I c w}^{c}+N \cdot \pi_{F}^{c}+N \cdot \sum_{x=1}^{\infty} \pi_{S n x}^{c} .
$$

Given these probabilities, we can derive the probabilities $p_{(j) i}^{c}$, needed to solve Node-State Markov chain, in which the channel is idle during $j$ 
successive backoff slots (but could have been more), for $1 \leq j \leq C W_{m}-1$, as follows:

$$
\begin{aligned}
p_{(j) i}^{c} & =p_{(j+1) i}^{c}+p_{b,(j) i}^{c} \\
& =p_{\left(C W_{m}\right) i}^{c}+\sum_{a=j}^{C W_{m}-1} p_{b,(a) i}^{c} .
\end{aligned}
$$

Finally, it should be noted that the tool which solves the model can be found at [14].

\subsection{Solving the case of study}

The Node-State Markov chain equations deployed in Appendix A depend on the initial parameters defined in the scenario and the conditional probabilities $p_{i \mid(j) i}^{c}$. For the case of study presented in 3.2 the initial param-

eters are the following: $p_{t}=0.0861$ (eqn. 1), $\mathbf{p}_{b}^{n 1-n 3}$ (eqn. 2), $M_{b o}^{n 1-n 3}$ and $C W^{n 1-n 3}$. The conditional probabilities $p_{i \mid(j) i}^{c}$ can be easily obtained numerically using a brute force algorithm in the following way: we guess a set of values for $p_{(j) i}^{c}$ for $j$ from 1 to $C W_{m}$ knowing that they must be between 1 and 0 and $p_{(j) i}^{c} \geq p_{(k) i}^{c}$ if $j<k$ and solve the Nodes-State Markov chains to obtain the conditional probabilities for the different class nodes to start a transmission, $p_{t \mid(m) i}^{n}$ (see eqn. 7). Knowing $p_{t \mid(m) i}^{n}$, it is possible to compute $\alpha_{j}$ and $\beta_{I j}^{n x}$ and solve the Channel-State equations. Once the Channel-State is solved the probability for the channel of being idle a certain amount of slots, $p_{(j) i}$ can be checked using eqn. 15 .

For the case of study only two conditional probabilities must be obtained by the brute force algorithm, this process may run for a few minutes in a general-purpose computer using Matlab, but the simulation time increases exponentially with $C W_{m}$. With $C W_{m}=3$ the simulation may run for a few hours and a metaheuristic approach, as simulated annealing or genetic algorithm, should be used to accelerate the convergence.

To solve the model for the case of study presented in 3.2, a resolution of 0.001 will be used for $p_{(j) i}^{c}$ and an error tolerance of 0.0005 will be set. Assuming a value of $p_{i}^{c}=0.2210$ and $p_{i i i}^{c}=0.0660$, this will lead us to $p_{i i}^{c}=0.1431, p_{i \mid i}^{c}=0.6475$, and $p_{i \mid i i}^{c}=0.4612$ according to eqn. 5 and 6 . Solving Appendix A equations for class-n1 node will give us the long-run proportions of transitions into the states, while using eqn. 3 we can obtain the channel access probability $p_{t}^{n 1}=0.0090$ and the conditional 
probability for a class-n1 node to start a transmission $p_{t \mid(2) i}^{n 1}=0.0629$ (eqn. 7). The same procedure can be used to obtain $p_{t \mid(2) i}^{n 2}=0.0651$ and $p_{t \mid(3) i}^{n 3}=0.1536$

The Channel-State Markov chain equations developed in Appendix B depend on the maximum contention window $C W_{m}=3$, the transition probabilities, $\boldsymbol{\alpha}$, and the transition probabilities to a Success state $\boldsymbol{\beta}$. As introduced in Section 3.2, $\alpha_{1}=1$, as no node can initiate a transmission while the channel has been idle for just one backoff slot. $\alpha_{2}$ represents the probability of no nodes of classes $\mathrm{n} 1$ and $\mathrm{n} 2$ begin a transmission (classn3 can not initiate the transmission as $C W^{n 3}=3$ ) an can be obtained by multiplying the non-zero conditional probabilities of starting a transmission $\alpha_{2}=\left(\bar{p}_{t \mid(2) i}^{n 1}\right)^{M_{n 1}} \cdot\left(\bar{p}_{t \mid(2) i}^{n 2}\right)^{M_{n 2}}=0.5892$ (eqn. 8). The last element will also include the class-n3 nodes $\alpha_{3}=\alpha_{2} \cdot\left(\bar{p}_{t \mid(3) i}^{n 3}\right)^{M_{n 3}}=0.3024$.

The transition probabilities $\boldsymbol{\beta}$ can be obtained using eqn. $9 . \beta_{I 1}^{n x}$ is 0 as no node can start a transmission form $B I_{1}$. From state $B I_{2}$, only nodes of classes $n 1$ and $n 2$ can successfully start a transmission, and so $\beta_{I 2}^{n 3}=0$. For n1 nodes the probability is $\beta_{I 2}^{n 1}=p_{t \mid(2) i}^{n 1} \cdot M_{n 1} \cdot\left(\bar{p}_{t \mid(2) i}^{n 1}\right)^{M_{n 1}-1} \cdot\left(\bar{p}_{t \mid(2) i}^{n 2}\right)^{M_{n 2}}=0.1581$, that is, only one class-n1 node starts a transmission while all n2 nodes do not attempt, and so the transmission is successful. For class-n2 is the opposite, just one class-n2 node begins a transmission while the rest of the nodes do not, leading $\beta_{I 2}^{n 2}=0.1641$. And finally, from $I_{3}$. From the point of view of class-n1, $\beta_{I 3}^{n 1}=\beta_{I 2}^{n 1} \cdot\left(\bar{p}_{t \mid(3) i}^{n 3}\right)^{M_{n 3}}=0.0811$, that is, also class-n3 nodes do not attempt to transmit. For class-n2 is similar $\beta_{I 3}^{n 2}=0.0842$. In case of class-n3 we have $\beta_{I 3}^{n 3}=0.2195$

Once we have the long-run proportions of transitions into the states for the Channel State Markov chain, and using eqn. 11 and 12, we obtain the conditional probabilities that will lead us to a new set of $p_{i}^{c}=0.2215$, $p_{i i}^{c}=0.1436$ and $p_{i i i}^{c}=0.0658$ using eqn. 15 that can be compared with the initial assumption giving a maximum absolute error of 0.0005 , equal to the error tolerance.

\section{Metrics Formulation}

Based on the resolution of the Node-State and Channel-State chains of our network, we are now ready to evaluate the most relevant metrics:

\subsection{Throughput}

The aggregate throughput $T h$ of the network is related to the percentage of time that the channel is in a Success state. This is given by the steady 
state probability of being in any success state of Figure 4 multiplied by the dwelling time, and can be derived to be:

$$
T h=\frac{N \cdot \sum_{j=1}^{\infty} \pi_{S n j}}{\sum C h S t} .
$$

In the case of study this value is $T h=0.5039$ which means that only $50 \%$ of the maximum bandwidth (BW) is efficiently spent. The absolutely maximum throughput for a standard IEEE 802.15.4 network with no ACK and a common packet length of $N$ is $\frac{N}{N+2}=0.83$ that means no collisions and only two idle bakoffs slots between packets.

For a class-n node, the throughput is given by the time spent in its corresponding Sucess state divided by the number of nodes in the group:

$$
T h_{n}=\frac{N \cdot \pi_{S n}}{M_{n} \cdot \sum C h S t},
$$

that give us $T h_{n} 1=0.0441, T h_{n} 2=0.0458$ and $T h_{n} 3=0.0361$. Class-n3 nodes have the least throughput, about $3.6 \%$ of the total transmit time that corresponds with an effective BW speed of $B W_{n 1}[K b p s]=T h_{n 1} \cdot 250[K b p s]$.

\subsection{Power Consumption}

The power consumption of the radio is related to each different state of the radio; we will define $P_{\text {idle }}$ as the mean power consumption during the idle state, $P_{t x}$ for transmitting and $P_{r x}$ for receiving. To compute these values, Ramachandran et.al. [8] model has been used. For the sake of completeness, the most relevant equations are included:

$$
\begin{aligned}
P_{i d l e} & =W_{i d l e} \cdot\left(\frac{\pi_{I D L E}^{n}+\sum_{j=1}^{C W^{n}} \pi_{B O j}^{n}}{\sum C h S t}-p_{b c n}-p_{i r}^{n}\right) \\
P_{t x} & =W_{t x} \cdot \frac{N \cdot \pi_{T X}^{n}}{\sum C h S t}, \\
P_{r x} & =W_{r x} \cdot\left(\frac{\sum_{j=1}^{M_{b o}^{n}} \sum_{j=1}^{C W^{n}} \pi_{C S j k}^{n}}{\sum C h S t}+p_{b c n}+p_{i r}^{n}\right)
\end{aligned}
$$

where $W_{i d l e}, W_{t x}$ and $W_{r x}$ are the power consumption of the radio in the different states $(0.712,31.32$ and $35.28 \mathrm{~mW}$ respectively will be used to illustrate the results, corresponding with the Chipcon cc2420 802.15.4 compliant 
$\mathrm{RF}$ transceiver [23]); $p_{b c n}$ is the probability of receiving a beacon during a backoff slot, computed as Poisson random variable a with a constant rate $\mu=B L / B I$ beacons per bakoff slot, (where $B L=2$ is the beacon length expressed in backoff slots and $B I=3072$ is the beacon interval in the same units):

$$
p_{b c n}=\frac{B L}{B I} \cdot e^{-B L / B I},
$$

and $p_{i r}^{n}$ is the probability of activating the radio due to a idle-receive transition and is equal to:

$$
p_{i r}^{n}=\frac{N_{i r}}{B I} \cdot \frac{\sum_{j=1}^{M_{b o}^{n}} \pi_{S C 1, j^{n}}}{\sum C h S t},
$$

where $N_{i r}$ is the activation time for cc 2420 , that is 0.6 backoff slots.

\subsection{Probability of Delivery}

The percentage of packets that reach the destination $p_{D}^{n}$ can be computed as:

$$
p_{D}^{n}=\left(1-p_{r e j}^{n}\right) \cdot p_{s e n d}^{n} \cdot P D R^{n},
$$

where $p_{r e j}^{n}$ is the probability of rejection, $p_{\text {send }}^{n}$ is the probability of sending (notice that not all sent packets reach the destination, a collision may occur) and $P D R^{n}$ is the Packet Delivery Ratio. No packet buffering is allowed, see Section 3.1 for details, and therefore a packet will be rejected with a probability $p_{r e j}^{n}$ if the node is not in the Idle state, that is given by:

$$
p_{r e j}^{n}=1-p_{i d l e}^{n},
$$

where $p_{i d l e}^{n}$ denotes the probability of a class-n node of being in the Idle state:

$$
p_{i d l e}^{n}=\frac{\pi_{I D L E}^{n}}{\sum N d S t^{n}} .
$$

The probability for an accepted packet to be sent over the wireless link, $p_{\text {send }}^{n}$, can be computed as the quotient of the dwelling time on $T X$ state and the total time the node is supposed to be in $T X$ state if it sends all the accepted packets:

$$
p_{\text {send }}^{n}=\frac{N \cdot \pi_{T X}^{n}}{\lambda \cdot \pi_{I D L E}^{n}} .
$$


And finally, the $P D R^{n}$ is defined as the quotient between the successfully received packets and the packets sent by the nodes and is equal to:

$$
P D R^{n}=\frac{T h_{n}}{M_{n}} \cdot p_{t}^{n}
$$

\subsection{Average Frame Service Time}

The average frame service time or latency, is defined as the average duration from the instant a packet becomes available for transmission to the end of its successful transmission. For a generic class-n node it is equal to the average duration between two successfully class-n transmission minus the average time spent by a class-n node in the $I D L E$ state during the latter duration:

$$
L^{n}=\frac{N \cdot M_{n}}{T h_{n}} \cdot\left(1-p_{i d l e}^{n}\right)
$$

\section{Model validation and performance results}

In this section we will present an analysis of the impact of modifying separately the different parameters of the CAP generic Markov-chain-based analytical model introduced in Section 3. Our goal is to study the effect of tuning the different parameters in order to provide traffic differentiation. In order to decouple the effect of the contention parameters in the metrics, we will modify separately each one. Notice that the Markov model presented in Section 5.1 can be used for scenarios in which more than 2 different classes of nodes share the channel, even using different data rates, and with different populations among the classes.

In Section 5.1 we present the default parameter scenario, 12 nodes with the same contention parameters equal to the default values specified for IEE 802.15.4 [15]. These results will be compared with three different scenarios in which the nodes will be equally distributed in two classes of nodes that share all the contention parameters but one, trying to isolate the effect of modifying one contention parameter on the metrics defined in Section 4.

Once the contention parameters have been studied separately, a specific scenario of high priority versus low priority class will be evaluated combining different parameter modification in Section 5.5. These results will be

presented along with the simulation results obtained using the $n s$ - 2 discrete 


\begin{tabular}{c|c|cccc|c|c}
\hline \hline Data rate & Thr. & \multicolumn{4}{|c|}{ Power } & $p_{D}^{n}$ & $L^{n}$ \\
{$[0-1]$} & {$[0-1]$} & Tot $[\mathrm{mW}]$ & $\operatorname{Tx}[\%]$ & $\operatorname{Rx}[\%]$ & Idle[\%] & {$[\%]$} & {$[$ backoffs] } \\
\hline$\lambda=0.01$ & 0.12 & 1.14 & 27.02 & 11.30 & 61.68 & 97.03 & 17.13 \\
$\lambda=0.05$ & 0.45 & 2.62 & 49.00 & 25.48 & 25.53 & 74.70 & 30.62 \\
$\lambda=0.90$ & 0.53 & 7.47 & 37.98 & 54.45 & 7.57 & 4.92 & 174.59 \\
\hline \hline
\end{tabular}

Table 1: Results of the default parameters scenario

event network simulator ${ }^{2}[13,24,25]$ in order to validate the mathematical model.

The data packets are 10 backoffs slots length and a data rate of $\lambda$ packets per packet duration will be set, therefore, the maximum data rate for a node is $\lambda=1$, in this case the node generates a 10 backoffs slots packet every $N=10$ backoffs. The goal will be the identification of suitable values for traffic differentiation among classes.

\subsection{Default parameters scenario}

Figure 5 and Figure 6 depict the comparison between Markov-Chain MAC generic model analytical results (solid lines) and the $n s-2$ simulator (dotted) for aggregate PAN throughput and energy consumption per node due to the radio for the default scenario. The maximum aggregate throughput is $T h=0.59$, that is, the channel is idle $41 \%$, and it is achieved when the packet rate is $\lambda=0.2$ packets per packet duration for each node (the theoretical maximum is $T h_{\max }=0.83$, see Section 4.1). It is also noticeable that for values above $\lambda=0.05$ the channel is saturated, that is, increasing $\lambda$ do not increase the packets delivered. In terms of radio energy consumption, the total power dissipation due to the wireless link is between $0.78 \mathrm{~mW}$ for low data rates, in which the highest contributor is the idle consumption, and up to about $7.58 \mathrm{~mW}$ when the channel is highly saturated. The reader should also notice that in the latter case, the nodes have the radio most of the time listening to the channel to avoid collision, and the radio uses even more power for performing the CSMA-CA than the power used for sending data.

Figure 7 and table 1 show statistical information that can be computed easily from the mathematical model, but can not be directly obtained

\footnotetext{
${ }^{2}$ Not all the metrics defined in Section 4 are available in $n s$ - 2 (by default just the throughput), and it is not possible to modify the contention parameters. Some modifications have been carried out in order to include the power consumption and the possibility of modifying the contention parameters
} 


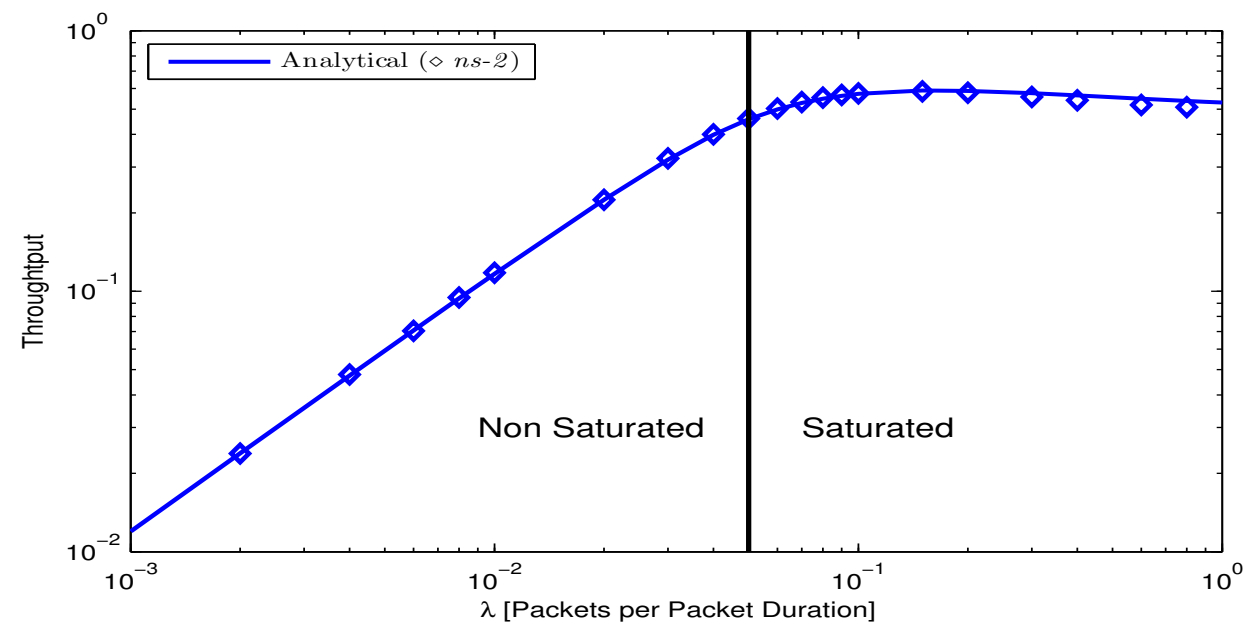

Figure 5: Throughput comparison of the Markov model (solid) and the ns-2 simulator (dots) for the default parameters scenario

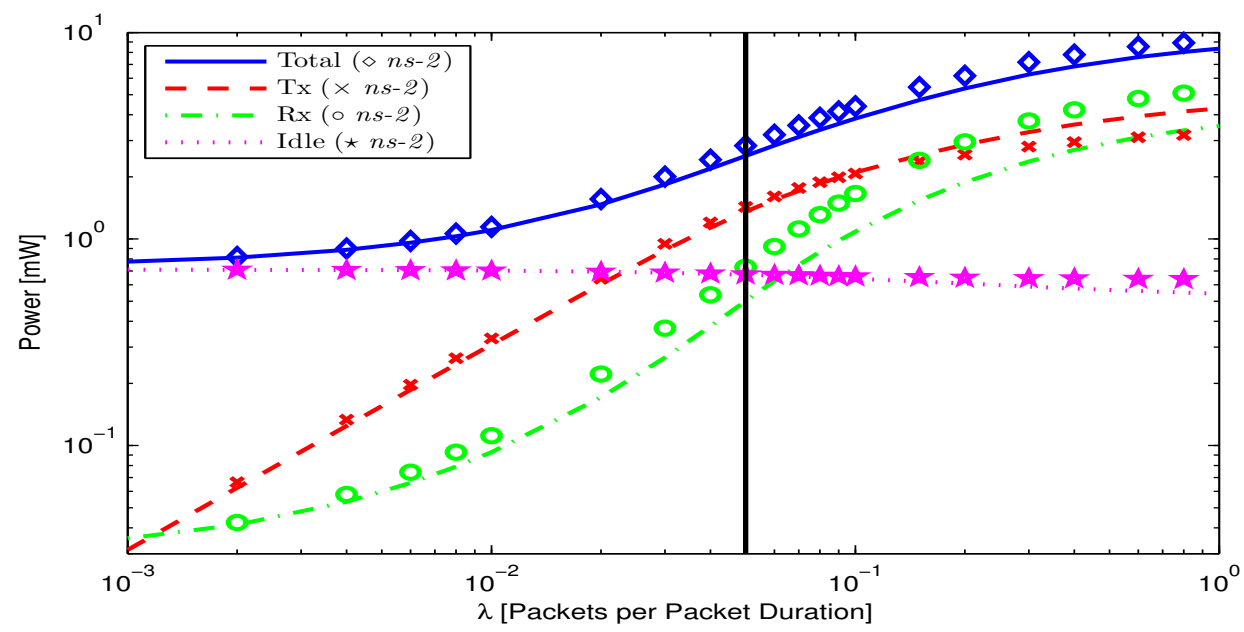

Figure 6: Power consumption due to Transmit, Receive and Idle states for the default parameters scenario

from $n s$ - 2 simulations. For example, the percentage of packets that reaches the destination, $p_{D}^{n}$, and the latency associated, $L^{n}$. For low data rates, $p_{D}^{n}\left(\lambda_{0.01}\right) \simeq 97 \%$, almost all packets are accepted and correctly sent with $L^{n}\left(\lambda_{0.01}\right) \simeq 17$ backoff slots, close to the absolute minimum of $1+N+C W=$ 13 , that is 1 for the transition to $C S_{1,1}$ plus sense the channel twice and finally 


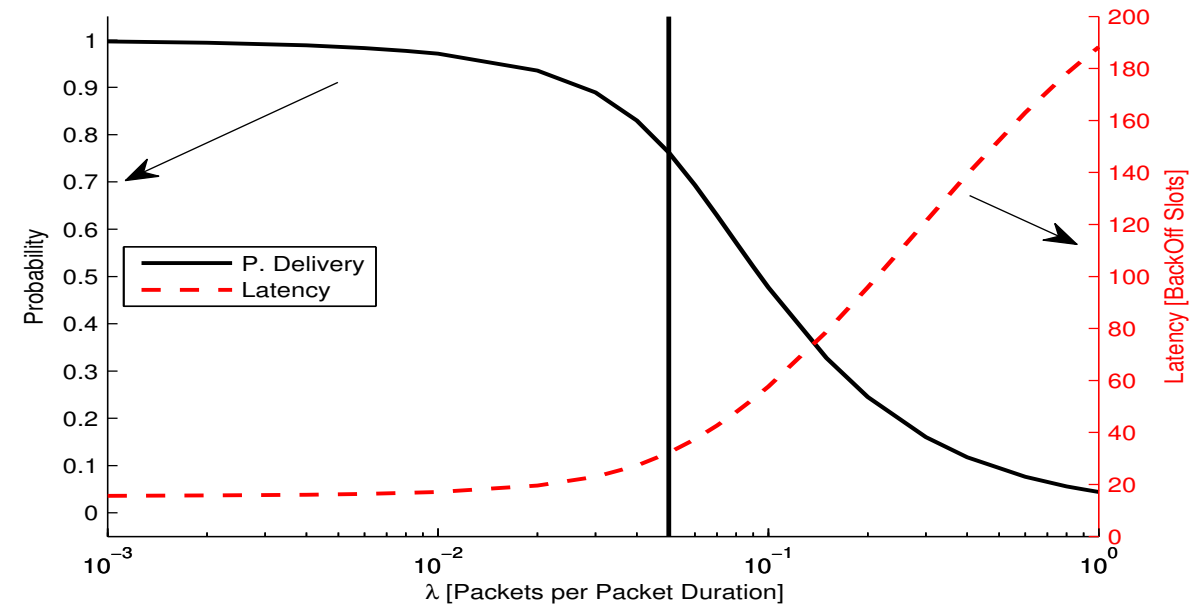

Figure 7: Probability of delivery and latency for the default parameters scenario

sending the packet. If we move $\lambda$ close to the saturation point, $p_{D}^{n}$ starts to decrease, $p_{D}^{n}\left(\lambda_{0.05}\right) \simeq 75 \%$, and the latency is also increased $L^{n}\left(\lambda_{0.05}\right) \simeq 31$, but if we move further into the saturation zone $p_{D}^{n}$ decreases dramatically $p_{D}^{n}\left(\lambda_{0.9}\right) \simeq 5 \%$ while the latency increases exponentially $L^{n}\left(\lambda_{0.9}\right) \simeq 175$, the system is unusable.

\subsection{Impact of a single parameter modification, $C W^{n}$}

Figure 8 shows the throughput of a scenario in which 12 nodes have been equally distributed in the different types of nodes, i.e., 6 class-n1 nodes with $C W^{n 1}=1$ and 6 class-n 2 nodes with $C W^{n 2}=2$. The other contention parameter values will be set to the default values specified by the 802.15.4 standard for both classes of nodes, that is $M_{b o}=4, \operatorname{macMinBE}=3$ and $\operatorname{macMax} B E=5$. The aggregate maximum throughput achieved is increased in comparison with the default parameters scenario, Section 5.1, the channel is being used $63 \%$ of the time when nodes are generating a traffic of $\lambda=$ 0.2 packets per packet duration. It is also noticeable how the channel is shared between the different types of nodes that access it. In this scenario class-n1 nodes have a better chance to deliver a packet in high saturation conditions, in comparison with class-n2, because they only have to sense the channel once before starting a transmission, having a $T h_{n 1}\left(\lambda_{0.9}\right) \simeq 41 \%$ versus $T h_{n 2}\left(\lambda_{0.9}\right) \simeq 19 \%$ for class-n2 nodes, that generates an aggregate throughput of $T h=60$, higher than the throughput obtained in the default 


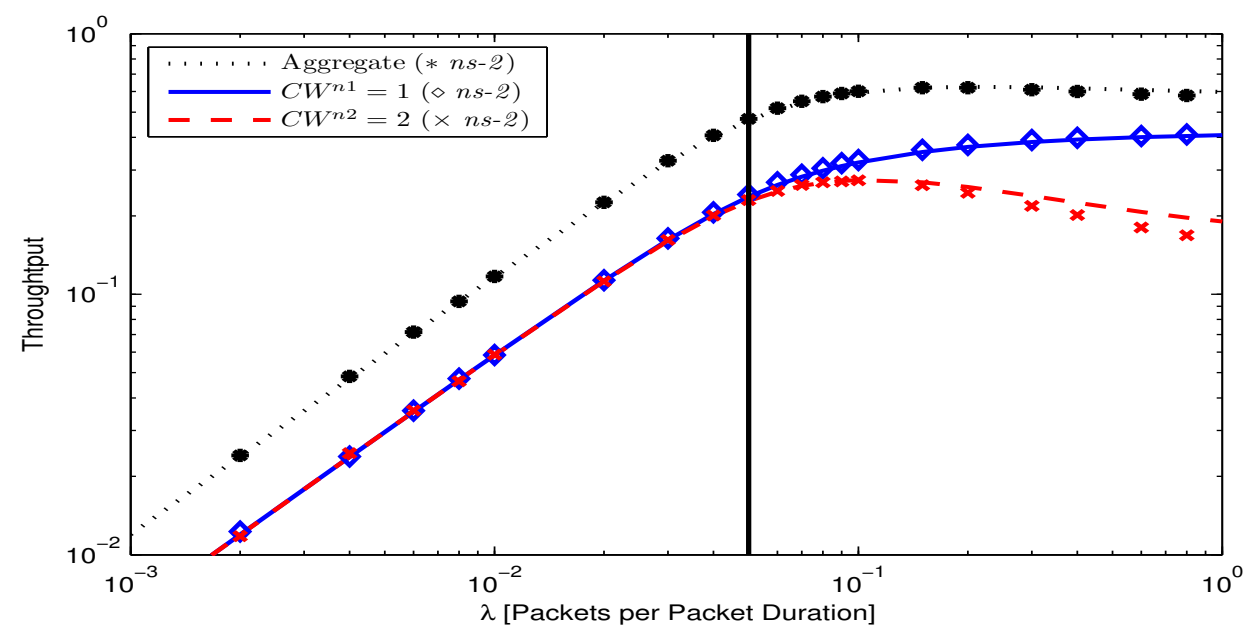

Figure 8: Throughput comparison for $C W^{n}$ variations $\left(C W^{n 1}=1, C W^{n 2}=2\right)$

scenario $\left(T h_{d e f}=53\right)$. It is also noticeable that the throughput for data rates below saturation point, $\lambda=0.05$, is almost identical for both classes of nodes, see table 2 .

In terms of energy consumption, the absolute values are very similar for class-n1 and $n 2$ in case of non saturated traffic (and a little less than the default parameter scenario), but in full saturated scenario class-n2 nodes spend most of the energy listening to the channel (61\%) while class-n1 distributes the energy consumption equally between transmission and listening.

In terms of latency, Figure 9 and Table 2 show how class-n2 nodes have a minimum of 17 backoff slots (as in the default scenario) while classn2 waits one backoff less, as expected, because they have to listen to the channel just once slot instead of two backoff slots. In the full saturated scenario $(\lambda=0.90)$ class-n1 have to wait 112 backoff slots $(\sim 11$ times the length of a packet and $36 \%$ less than the default scenario) while class-n2 nodes wait up to 244 backoff slots ( $\sim 24$ times the length of a packet and $39 \%$ more than the default scenario).

We can conclude that in scenarios with low saturated traffic, the two populations of nodes behave in the same way, sending correctly all the packets with a low latency, but if we move to a scenario with high saturated traffic, nodes that need to sense the channel during more backoff slots will have their bandwidth reduced and their latency increased significantly in favour of nodes with a lower contention window, and so $C W^{n}$ can be used for 


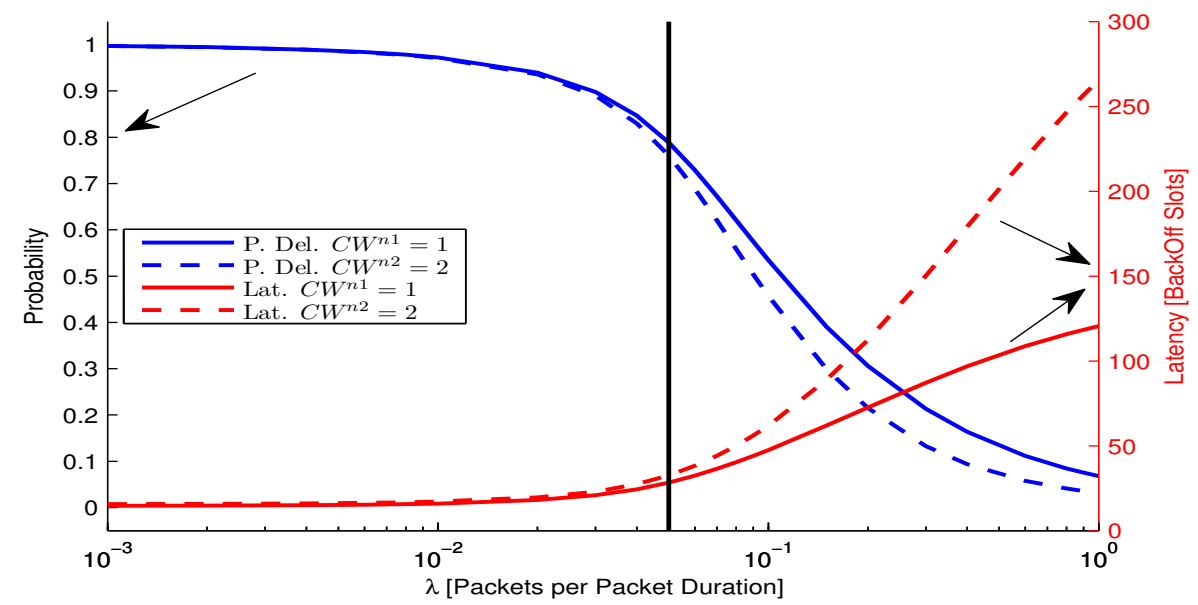

Figure 9: Probability of deliver and Latency for $C W^{n}$ variations $\left(C W^{n 1}=1\right.$, $C W^{n 2}=2$ )

\begin{tabular}{|c|c|c|c|c|c|c|c|c|}
\hline \multirow{2}{*}{ Class } & \multirow{2}{*}{$\begin{array}{c}\text { Data rate } \\
{[0-1]}\end{array}$} & \multirow{2}{*}{$\begin{array}{l}\text { Thr. } \\
{[0-1]}\end{array}$} & \multicolumn{4}{|c|}{ Power } & \multirow{2}{*}{$\begin{array}{l}p_{D}^{n} \\
{[\%]}\end{array}$} & \multirow{2}{*}{$\begin{array}{c}L^{n} \\
{[\text { backoffs] }}\end{array}$} \\
\hline & & & $\operatorname{Tot}[\mathrm{mW}]$ & $\mathrm{Tx}[\%]$ & $\mathrm{Rx}[\%]$ & Idle[\%] & & \\
\hline \multirow{3}{*}{$C W^{n 1}=1$} & $\lambda=0.01$ & 0.06 & 1.10 & 27.91 & 8.40 & 63.69 & 97.16 & 15.95 \\
\hline & $\lambda=0.05$ & 0.23 & 2.48 & 53.36 & 19.59 & 27.06 & 77.55 & 27.14 \\
\hline & $\lambda=0.90$ & 0.41 & 7.69 & 46.65 & 46.08 & 7.26 & 7.53 & 112.33 \\
\hline \multirow{3}{*}{$C W^{n 2}=2$} & $\lambda=0.01$ & 0.06 & 1.14 & 27.02 & 11.30 & 61.68 & 97.03 & 17.13 \\
\hline & $\lambda=0.05$ & 0.22 & 2.62 & 48.77 & 25.68 & 25.56 & 74.41 & 31.01 \\
\hline & $\lambda=0.90$ & 0.19 & 6.74 & 30.69 & 60.67 & 8.64 & 3.56 & 243.81 \\
\hline
\end{tabular}

Table 2: Impact of $C W^{n}$ variations

traffic differentiation.

\subsection{Impact of a single parameter modification, $M_{b o}^{n}$}

Figure 10 shows the throughput in case of 2 different types of nodes, 6 nodes with $M_{b o}^{n}=1$ and 6 with $M_{b o}^{n}=5$. The aggregate throughput in this scenario is almost identical to the default parameters scenario $59 \%$, but in moderate saturation scenario, $\lambda \approx 0.05$, nodes with $M_{b o}^{n}=1$ will obtain a lower throughput than nodes with $M_{b o}^{n}=5$ because they only try once to send a packet, and therefore they have a high probability of failing, see Figure 11. But when increasing $\lambda$ into high saturation mode, the throughput for $M_{b o}^{n}=1$ nodes goes up but decreases for $M_{b o}^{n}=5$. The reason of that effect lies on the fact that the probability of backing off in the first backoff stage, $\bar{p}_{b 1}^{n}$ on Figure 3, is lower than backing off in other stages for a default $\mathbf{p}_{b}^{n}$, and as both types of nodes are always trying to send a packet in a full saturation 


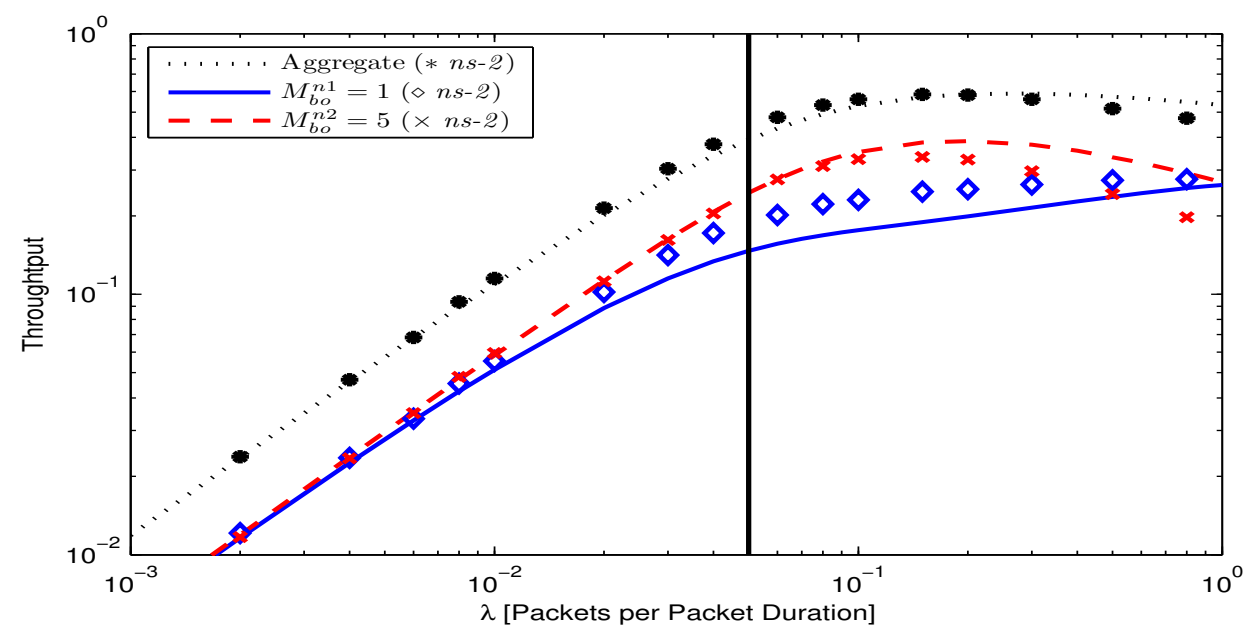

Figure 10: Throughput comparison for $M_{b o}^{n}$ variations $\left(M_{b o}^{n 1}=1, M_{b o}^{n 2}=5\right)$

scenario, nodes using $M_{b o}^{n}=1$ will have a lesser probability of backing-off before starting sensing the channel, and this effect will compensate the higher chance of failing sending a packet.

In terms of energy consumption, see Table 3, both nodes perform very similar and their values are also comparable with the values obtained in the default parameter scenario.

It must also be noticed that the differences between the generic MAC model and the $n s-2$ simulation have been increased, but show a similar fashion. This is due to the extreme situation for nodes using only one backoff stage, $M_{b o}^{n}=1$. This effect is attenuated when a bigger $M_{b o}^{n}$ is used, but the effect of this parameter in the metrics is very limited in that situations, and therefore will not be studied.

Figure 11 depicts the probability of delivery and latency for both types of nodes. In comparison with previous results, see Figure 9, the differentiation between the two node types has been increased. For $M_{b o}^{n}=1$, the probability of deliver starts decreasing and the latency increases for lower values of $\lambda$, notice that $p_{D}^{n 1}(\lambda=0.05)<50 \%$, see Table 3 .

We can conclude that even in scenarios with low saturated traffic, using a very small value for $M_{b o}^{n}=1$ will produce a very poor performance in terms of throughput and packet delivery ratio, while increasing its value above a certain level $\left(M_{b o}^{n}>3\right)$ has a very limited impact on the system, and so it is not very useful for traffic differentiation. 


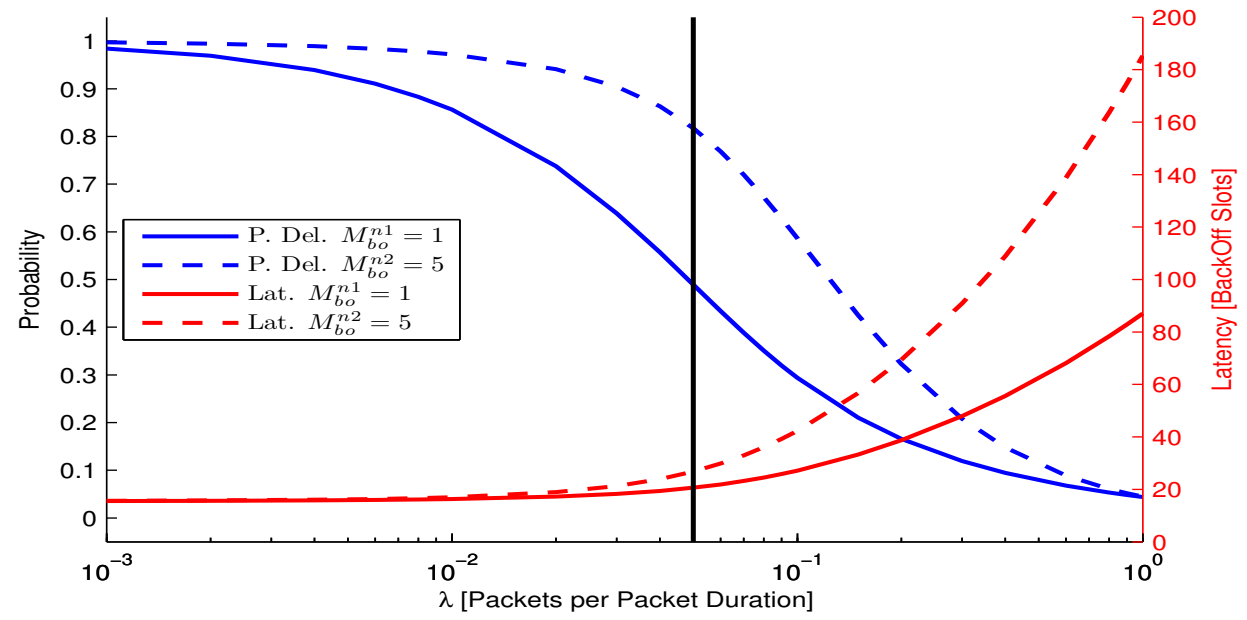

Figure 11: Probability of deliver and Latency for $M_{b o}^{n}$ variations $\left(M_{b o}^{n 1}=1, M_{b o}^{n 2}=\right.$ 5)

\begin{tabular}{|c|c|c|c|c|c|c|c|c|}
\hline \multirow{2}{*}{ Class } & \multirow{2}{*}{$\begin{array}{c}\text { Data rate } \\
{[0-1]}\end{array}$} & \multirow{2}{*}{$\begin{array}{l}\text { Thr. } \\
{[0-1]}\end{array}$} & \multicolumn{4}{|c|}{ Power } & \multirow{2}{*}{$\begin{array}{l}p_{D}^{n} \\
{[\%]}\end{array}$} & \multirow{2}{*}{$\begin{array}{c}L^{n} \\
\text { [backoffs] }\end{array}$} \\
\hline & & & $\operatorname{Tot}[\mathrm{mW}]$ & $\operatorname{Tx}[\%]$ & $\mathrm{Rx}[\%]$ & Idle[\%] & & \\
\hline \multirow{3}{*}{$M_{b o}^{n 1}=1$} & $\lambda=0.01$ & 0.05 & 1.09 & 24.86 & 10.67 & 64.47 & 85.60 & 16.33 \\
\hline & $\lambda=0.05$ & 0.15 & 1.90 & 43.19 & 20.83 & 35.98 & 48.90 & 20.61 \\
\hline & $\lambda=0.90$ & 0.26 & 6.85 & 38.37 & 53.18 & 8.45 & 4.78 & 81.12 \\
\hline \multirow{3}{*}{$M_{b o}^{n 2}=5$} & $\lambda=0.01$ & 0.06 & 1.14 & 27.04 & 11.25 & 61.70 & 97.14 & 17.02 \\
\hline & $\lambda=0.05$ & 0.25 & 2.67 & 51.12 & 23.91 & 24.97 & 81.54 & 26.76 \\
\hline & $\lambda=0.90$ & 0.28 & 7.43 & 38.73 & 53.65 & 7.62 & 5.26 & 170.51 \\
\hline
\end{tabular}

Table 3: Impact of $M_{b o}^{n}$ variations

\subsection{Impact of a single parameter modification, $\mathbf{p}_{b}^{n}$ $\left(\operatorname{macMinB} E^{n}-\operatorname{macMax} B E^{n}\right)$.}

Figures 12 and 13 show the throughput, probability of delivery and latency in case of 2 different types of nodes, 6 nodes with $\mathbf{p}_{b}^{n 1}$ obtained from $\operatorname{macMin} B E^{n 1}=0, \operatorname{macMax} B E^{n 1}=5$, that will be denoted in the figures as $\mathbf{p}_{b}^{n 1}=[0,5]$, and 6 with $\mathbf{p}_{b}^{n 2}=[3,5]\left(\operatorname{macMin} B E^{n 2}=3, \operatorname{macMax} B E^{n 2}=\right.$ $5)$. The throughput differentiation obtained in this scenario is similar to the case of using $C W^{n}$ to differentiate nodes, but with a lesser aggregate value and a significant higher absolute energy consumption for class-n1 in full saturated mode, see Table 2 and Table 4 . When $\mathbf{p}_{b}^{n 1}$ is used, a higher throughput is obtained due to the lesser time spent in the backoff stages in comparison to using $\mathbf{p}_{b}^{n 2}$, but it affects the energy as class-n1 nodes do not separate their attempts of sending a packet in the initial backoff stages. 


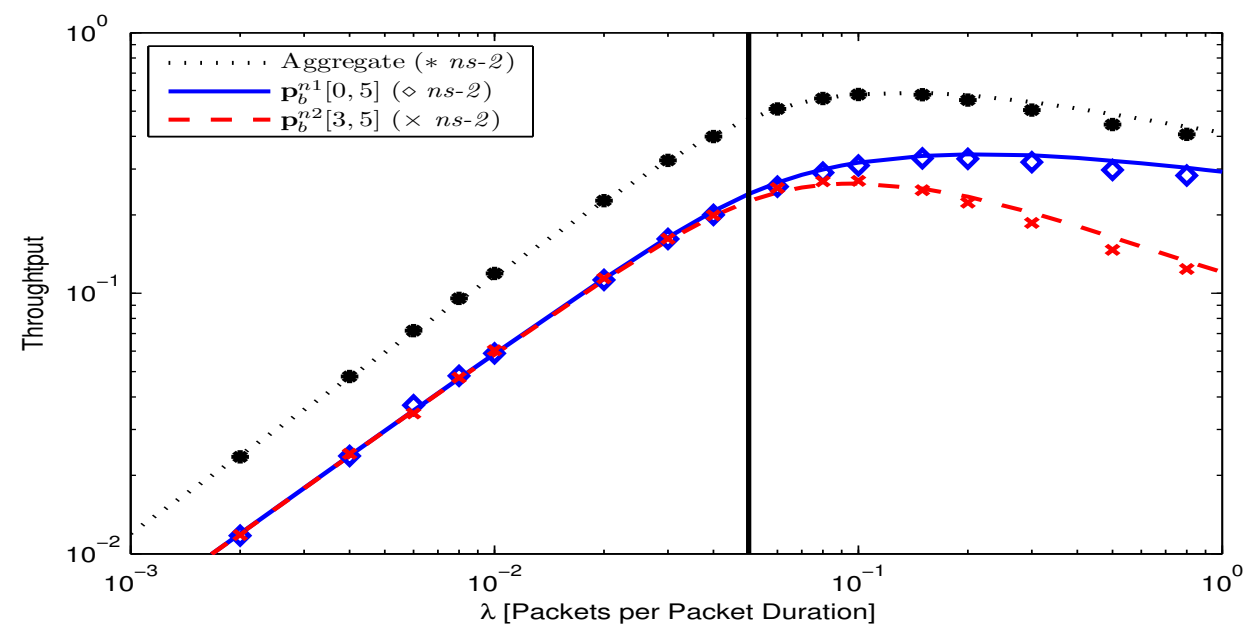

Figure 12: Throughput comparison for $\mathbf{p}_{b}^{n}$ variations $\left(\mathbf{p}_{b}^{n 1}=[0,5], \mathbf{p}_{b}^{n 2}=[3,5]\right)$

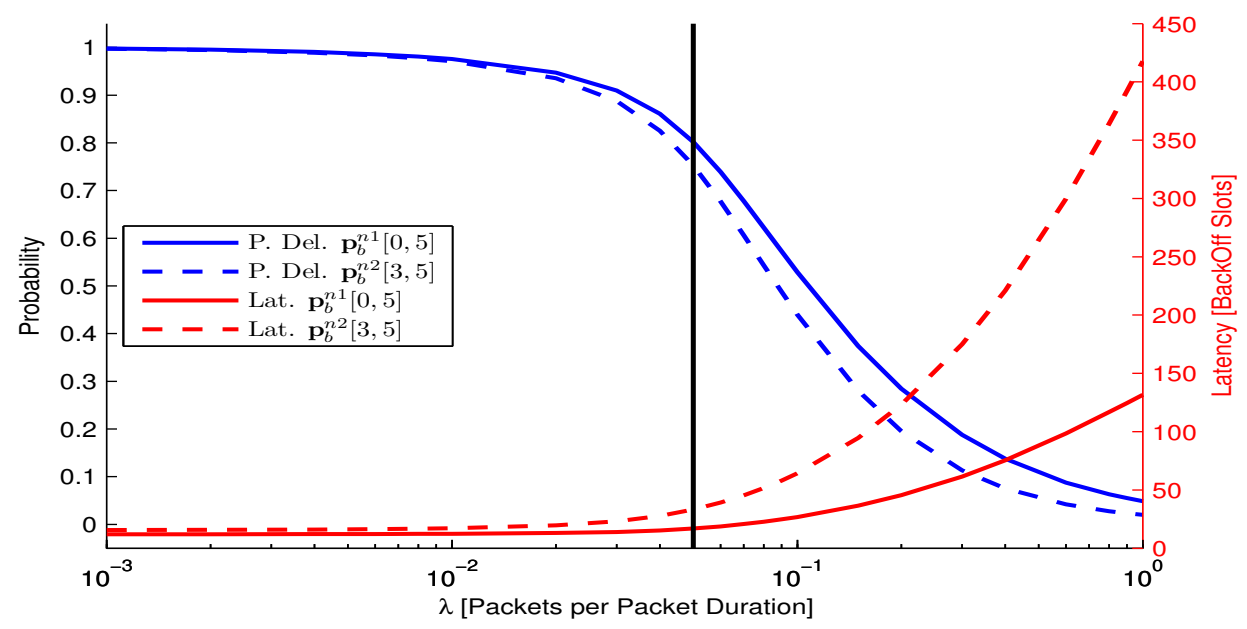

Figure 13: Probability of deliver and Latency for $\mathbf{p}_{b}^{n}$ variations $\left(\mathbf{p}_{b}^{n 1}=[0,5]\right.$, $\left.\mathbf{p}_{b}^{n 2}=[3,5]\right)$

The probability of delivery, Figure 13, is also very similar to the one obtained when changing $C W^{n}$, but a higher differentiation in terms of latency is obtained, going up to 420 backoffs in case of using $\mathbf{p}_{b}^{n 2}$.

We can conclude that $\mathbf{p}_{b}^{n}$ is also suitable for traffic differentiation. In the next section we will combine $C W^{n}$ and $\mathbf{p}_{b}^{n}$ to obtain a high priority class in comparison with a standard node. 


\begin{tabular}{|c|c|c|c|c|c|c|c|c|}
\hline \multirow{2}{*}{ Class } & \multirow{2}{*}{$\begin{array}{c}\text { Data rate } \\
{[0-1]}\end{array}$} & \multirow{2}{*}{$\begin{array}{l}\text { Thr. } \\
{[0-1]}\end{array}$} & \multicolumn{4}{|c|}{ Power } & \multirow{2}{*}{$\begin{array}{l}p_{D}^{n} \\
{[\%]}\end{array}$} & \multirow{2}{*}{$\begin{array}{c}L^{n} \\
\text { [backoffs] }\end{array}$} \\
\hline & & & $\operatorname{Tot}[\mathrm{mW}]$ & $\operatorname{Tx}[\%]$ & $\mathrm{Rx}[\%]$ & Idle[\%] & & \\
\hline \multirow{3}{*}{$\mathbf{p}_{b}^{n 1}=[0,5]$} & $\lambda=0.01$ & 0.06 & 1.14 & 27.11 & 11.32 & 61.57 & 97.46 & 12.42 \\
\hline & $\lambda=0.05$ & 0.24 & 2.80 & 49.61 & 26.64 & 23.75 & 80.02 & 16.92 \\
\hline & $\lambda=0.90$ & 0.30 & 13.75 & 34.54 & 62.33 & 3.14 & 5.55 & 121.67 \\
\hline \multirow{3}{*}{$\mathbf{p}_{b}^{n 2}=[3,5]$} & $\lambda=0.01$ & 0.06 & 1.14 & 27.03 & 11.30 & 61.67 & 97.02 & 17.14 \\
\hline & $\lambda=0.05$ & 0.23 & 2.68 & 48.78 & 26.26 & 24.96 & 75.12 & 33.08 \\
\hline & $\lambda=0.90$ & 0.13 & 6.78 & 32.52 & 58.92 & 8.57 & 2.38 & 381.82 \\
\hline
\end{tabular}

Table 4: Impact of $\mathbf{p}_{b}^{n}\left(\operatorname{macMin} B E^{n}-\operatorname{macMax} B E^{n}\right)$ variations

\subsection{Adding two effects, $C W^{n}$ and $\mathbf{p}_{b}^{n}$ (macMinB $\left.E^{n}-\operatorname{macMax} B E^{n}\right)$ for traffic differentiation.}

As it has been described in Sections 5.2 and 5.4,CW $W^{n}$ and $\mathrm{p}_{b}^{n}$ can be used to increase the throughput and reduce the latency of some nodes in comparison with others. These effects can be added to increase the throughput and latency discrimination. Figures 14 and 15 show the throughput, probability of delivery and latency in case of 2 different types of nodes, 6 nodes with $C W^{n 1}=1 \& \mathbf{p}_{b}^{n 1}=[0,5]$ and 6 with $C W^{n 2}=2 \& \mathbf{p}_{b}^{n 2}=[3,5]$ (default IEEE 802.15.4 parameters). The maximum aggregate throughput $4 \%$ higher than the default parameter scenario, that is $63 \%$ of the maximum when the packet rate is $\lambda=0.15$ in comparison with $59 \%$. It must be noticed that in this scenario almost all the bandwidth for high saturation mode is used by class-n1 nodes, having also a very low latency associated, making this set up suitable for implementing channel access prioritization.

If we compare class-n1 nodes, Table 5 , with the default parameter scenario, Table 1, we can see how class-n1 has a prioritized access to the channel in a full saturated scenario, the probability of delivery, $p_{D}^{n}$, is increased by a $77 \%$, it goes from $4.92 \%$ to $8.72 \%$, while the latency, $L^{n}$, is reduced in a $56 \%$, from 174.59 to 75.66 backoff slots, while the energy consumption is increased in a $83 \%$, form 7.47 to $13.68 \mathrm{~mW}$. In the other hand, class-n2 is highly penalised, with a throughput and $p_{D}^{n}$ dramatically reduced and the latency severely increased.

We can conclude that, in high saturation scenario $C W^{n}$ and $\mathbf{p}_{b}^{n}$ are a very effective parameters to be tuned in order to obtain traffic differentiation between different classes of nodes.

\section{Conclusions}

An Markov-chain-based analytical model has been introduced to evaluate the impact of the relevant IEEE 802.15.4 CAP parameters, namely: 


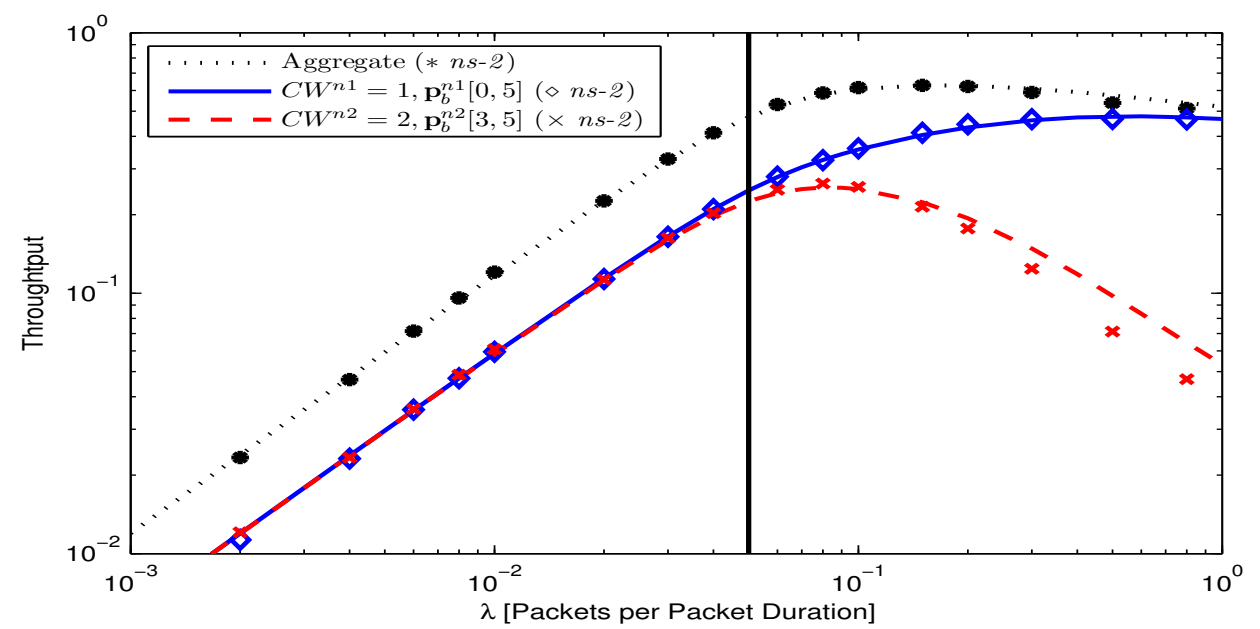

Figure 14: Throughput comparison of adding two effects $\left(C W^{n 1}=1 \& \mathbf{p}_{b}^{n 1}=[0,5]\right.$, $\left.C W^{n 2}=2 \& \mathbf{p}_{b}^{n 2}=[3,5]\right)$

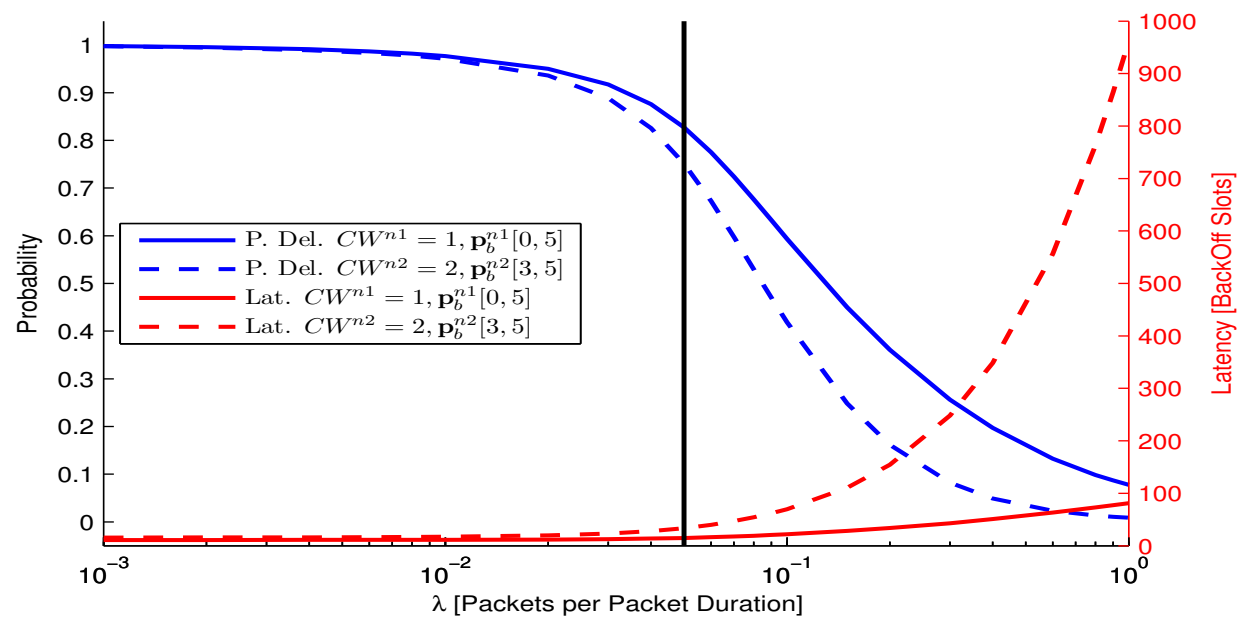

Figure 15: Probability of deliver and Latency of adding two effects $\left(C W^{n 1}=1\right.$ $\left.\& \mathbf{p}_{b}^{n 1}=[0,5], C W^{n 2}=2 \& \mathbf{p}_{b}^{n 2}=[3,5]\right)$

throughput, energy consumption, probability of delivering a packet and latency. This model has been implemented to evaluate several cases of study and it is publicly available. Its accuracy has been compared with the $n s-2$ discrete event network simulator, achieving good results.

Our model has proved to be a guide in the CAP configuration, in order to 


\begin{tabular}{c|c|c|cccc|c|c}
\hline \hline \multirow{2}{*}{ Class } & Data rate & Thr. & \multicolumn{4}{|c|}{ Power } & $p_{D}^{n}$ & $L^{n}$ \\
& {$[0-1]$} & {$[0-1]$} & $\operatorname{Tot}[\mathrm{mW}]$ & $\mathrm{Tx}[\%]$ & $\mathrm{Rx}[\%]$ & Idle[\%] & {$[\%]$} & {$[$ backoffs] } \\
\hline \multirow{3}{*}{$C W^{n 1}=1, \mathbf{p}_{b}^{n 1}=[0,5]$} & $\lambda=0.01$ & 0.06 & 1.11 & 28.00 & 8.41 & 63.59 & 97.58 & 11.36 \\
& $\lambda=0.05$ & 0.25 & 2.63 & 54.09 & 20.48 & 25.44 & 82.53 & 14.89 \\
& $\lambda=0.90$ & 0.47 & 13.68 & 41.73 & 55.12 & 3.14 & 8.72 & 75.66 \\
\hline \multirow{2}{*}{$C W^{n 2}=2, \mathbf{p}_{b}^{n 2}=[3,5]$} & $\lambda=0.01$ & 0.06 & 1.14 & 27.03 & 11.30 & 61.67 & 97.02 & 17.14 \\
& $\lambda=0.05$ & 0.23 & 2.68 & 48.58 & 26.45 & 24.97 & 75.02 & 33.45 \\
& $\lambda=0.90$ & 0.06 & 5.65 & 18.26 & 71.00 & 10.74 & 1.12 & 826.01 \\
\hline \hline
\end{tabular}

Table 5: Impact of adding two effects, $C W^{n}$ and $\mathbf{p}_{b}^{n}\left(\operatorname{macMin} B E-\operatorname{macMax} B E^{n}\right)$

properly select the parameter values for different classes of nodes, which are typically deployed in single-hop WSNs to obtain traffic differentiation. In case of high saturation scenario, the CAP parameters can be tuned to give $77 \%$ more chances of correctly sending a packet and $56 \%$ latency reduction (with $84 \%$ energy penalty) in comparison with the standard IEEE 802.15.4 CAP scenario.

Finally, we would like to pinpoint what directions are being followed to complement this work in the early future. On the one hand, the combination of the Markov-chain-based analytical model with a queue system is being considered. The basic idea consists of avoiding packet discarding in low/moderate traffic saturation mode. And on the other hand, several scenarios where the nodes dynamically modify their contention parameters are being studied. This is a promising line that may help the nodes accomodate their needs.

\section{APPENDIXES}

\section{Appendix A. Steady State Transition Equations For A Generic Class-n Markov Chain Model}

To obtain the steady-state probabilities of the embedded Markov chain for a generic class-n node the following balance equations must be solved. 


$$
\begin{aligned}
\pi_{I D L E}^{n}= & \sum_{j=1}^{C W^{n}}\left(\bar{p}_{i \mid(j-1) i}^{c} \cdot \pi_{C S m_{b o}^{n}, j}^{n}\right)+\pi_{T X}^{n}+\bar{p}_{t} \cdot \pi_{I D L E}^{n}, \\
\pi_{B O 1}^{n}= & p_{t} \cdot \bar{p}_{b 1}^{n} \cdot \pi_{I D L E}^{n}+\bar{p}_{b 1}^{n} \cdot \pi_{B O_{1}}^{n}, \\
\pi_{C S 1,1}^{n}= & p_{t} \cdot p_{b 1}^{n} \cdot \pi_{I D L E}^{n}+p_{b 1}^{n} \cdot \pi_{B O 1}^{n} \\
& C W^{n} \\
\pi_{B O j}^{n}= & \sum_{k=1}\left(\bar{p}_{i \mid(k-1) i}^{c} \cdot \bar{p}_{b j}^{n} \cdot \pi_{C S j-1, k}^{n}\right)+\bar{p}_{b j}^{n} \cdot \pi_{B O j}^{n}, \quad 2 \leq j \leq M_{b o}^{n}, \\
\pi_{C S j, 1}^{n}= & \sum_{k=1}^{C W^{n}}\left(\bar{p}_{i \mid(k-1) i}^{c} \cdot p_{b j}^{n} \cdot \pi_{C S j-1, k}^{n}\right)+p_{b j}^{n} \cdot \pi_{B O}^{n}, \quad 2 \leq j \leq M_{b o}^{n}, \\
\pi_{C S j, k}^{n}= & p_{i \mid(k-2) i}^{c} \cdot \pi_{C S j, k-1}^{n}, \quad 1 \leq j \leq M_{b o}^{n} \wedge 2 \leq k \leq C W^{n}, \\
\pi_{T X}^{n}= & 1-\pi_{I D L E}^{n}-\sum_{j=1}^{M_{b o}^{n}} \pi_{B O j}^{n}-\sum_{j=1}^{M_{b o}^{n}} \sum_{k=1}^{C W^{n}} \pi_{C S j, k}^{n} .
\end{aligned}
$$

\section{Appendix B. Steady State Transition Equations For The Shared Channel}

With respect to the steady-state probabilities of the embedded Markov chain depicted on Figure 4 the following balance equations must be solved:

$$
\begin{aligned}
\pi_{B I 1}^{c} & =\pi_{F}^{c}+\sum_{j=1}^{\infty} \pi_{S n j} \\
\pi_{B I j}^{c} & =\alpha_{j-1} \cdot \pi_{B I j-1}^{c}, \quad 2 \leq j<C W_{m}-1, \\
\pi_{I c w}^{c} & =\alpha_{c w} \cdot \pi_{B I c w-1}^{c}+\alpha_{c w} \cdot \pi_{I c w}^{c} \\
\pi_{S n j}^{c} & =\beta_{j, k}^{W} \cdot \pi_{(W) i}+\sum_{a=j}^{W-1}\left(\beta_{j, k}^{a} \cdot \pi_{b,(a) i}\right), \quad 2 \leq j<\infty, \\
\pi_{F} & =1-\pi_{(W) i}^{c}-\sum_{a=1}^{W-1} \pi_{b,(a) i}^{c}-\sum_{a=1}^{W} \sum_{b=1}^{\infty} \pi_{s c_{a, b}} .
\end{aligned}
$$

\section{Acknowledgements}

The authors would like to thank Eugene David Ngangue Ndih for his technical contributions to this project. 


\section{References}

1. Kleinrock, L., Tobagi, F.. Packet switching in radio channels: Part I-carrier sense multiple-access modes and their throughput-delay characteristics. IEEE Trans on Communications 1975;23:1400-1416.

2. Zheng, J., Lee, M.J.. Will IEEE 802.15.4 make ubiquitous networking reality? - a discussion on a potential low power, low bit rate standard. IEEE Communications Magazine 2004;42:140-146.

3. Koubaa, A., Alves, M., Tovar, E.. A comprehensive simulation study of slotted CSMA/CA for IEEE 802.15.4 wireless sensor networks. In: Proc. of IEEE International Workshop on Factory Communication Systems. 2006:183-192.

4. Bianchi, G.. Performance analysis of the IEEE 802.11 distributed coordination function. IEEE Journal on Selected Areas in Communications 2000;18(3):535-547.

5. Pollin, S., Ergen, M., Ergen, S., Bougard, B., Der Perre, L., Moerman, I., Bahai, A., Varaiya, P., Catthoor, F.. Performance analysis of slotted carrier sense ieee 802.15.4 medium access layer. IEEE Transactions on Wireless Communications 2008;7(9):3359 -3371. doi:10.1109/TWC.2008.060057.

6. Patro, R.K., et al., . Analysis and improvement of contention access protocol in IEEE 802.15.4 star network. In: Proc. of IEEE Conference on Mobile Adhoc and Sensor Systems (MASS). 2007:1-8.

7. Jung, C., Hwang, H., Sung, D.K., Hwang, G.. Enhanced markov chain model and throughput analysis of the slotted csma/ca for ieee 802.15.4 under unsaturated traffic conditions. IEEE Transactions on Vehicular Technology 2009;58(1):473-478.

8. Ramachandran, I., Das, A.K., Roy, S.. Analysis of the contention access period of IEEE 802.15.4. ACM Trans on Sensor Networks 2007;3(1):article 4.

9. Ling, X., et al., . A renewal theory based analytical model for the contention access period of IEEE 802.15.4. IEEE Trans on Wireless Communications 2008;7(6):2340-2349. 
10. Ndih, E., Khaled, N., Micheli, G.D.. An analytical model for the contention access period of the slotted ieee 802.15.4 with service differentiation. In: Proc. of IEEE International Conference on Communications ICC. 2009:1-6. doi:10.1109/ICC.2009.5198719.

11. Kim, E., Kim, M., Youm, S.K., S. Choi, C.H.K.. Multi-level service differentiation scheme for the IEEE 802.15.4 networks. In: Proc. of EUC Workshops. 2005:693-703.

12. Zhu, H., Chlamtac, I.. Performance analysis for IEEE 802.11e EDCF service differentiation. IEEE Trans on Wireles Communications 2005;4(4):1780-1788.

13. ns-2, . Chipcon products from texas intruments. 2010. URL: http://nsnam.isi.edu/nsnam/index.php.

14. Recas, J.. Cases of study for markov generic model of 802.15.4 CAP. 2013. URL: http://code.google.com/p/markov-802-15-4/.

15. Part 15.4: Wireless medium access control (MAC) and physical layer (PHY) specifications for low-rate wireless personal area networks (LRWPANs). Tech. Rep. IEEE 802.15.4 Std.; IEEE; 2006.

16. Stewart, J., Stewart, R., Hassan, M.N., Allen, J.. Application of decay rate analysis for gts provisioning in wireless sensor networks. In: Proc. of 8th International Symposium on Communication Systems, Networks 85 Digital Signal Processing (CSNDSP). IEEE; 2012:1-6.

17. Li, C., Li, H.B., Kohno, R.. Performance evaluation of ieee 802.15. 4 for wireless body area network (wban). In: Proc. of IEEE International Conference on Communications Workshops. IEEE; 2009:1-5.

18. Li, X., Hunter, D.. Analytical model of single-hop ieee 802.15. 4 data aggregation in wireless sensor networks. In: Proc. of IEEE International Conference on Communications Workshops. IEEE; 2009:1-6.

19. Kannan, L.N., Agarwal, N., Fumagalli, A., Tacca, M.. A markov chain model to account for multi-rate transmission and node cooperative behavior in ieee 802.11 data link protocol. In: Proc. of IEEE International Symposium on Modeling, Analysis and Simulation of Computers and Telecommunication Systems. IEEE; 2008:1-10. 
20. Al-Anbagi, I., Erol-Kantarci, M., Mouftah, H.. A reliable ieee 802.15.4 model for cyber physical power grid monitoring systems. IEEE Transactions on Emerging Topics in Computing 2013;PP(99):1-1. doi:10.1109/TETC.2013.2281192.

21. He, J., Tang, Z., Chen, H.H., Zhang, Q.. An accurate and scalable analytical model for ieee 802.15. 4 slotted csma/ca networks. IEEE Transactions on Wireless Communications 2009;8(1):440-448.

22. He, J., Tang, Z., Chen, H.H., Wang, S.. An accurate markov model for slotted csma/ca algorithm in ieee 802.15. 4 networks. IEEE Communications Letters 2008;12(6):420-422.

23. cc2420 2.4GHz IEEE 802.15.4 RF Transceiver, . Discrete network simulator. 2010. URL: http://www.ti.com/.

24. Charfi, F., Bouyahi, M.. Performance evaluation of beacon enabled ieee 802.15. 4 under ns2. International Journal of Distributed and Parallel Systems 2012;.

25. Edwards, J., Demers, F., St-Hilaire, M., Kunz, T.. Comparison of ns2. 34's zigbee/802.15. 4 implementation to memsic's iris motes. In: Proc. of rth International Wireless Communications and Mobile Computing Conference (IWCMC). IEEE; 2011:986-991. 